\title{
Thermal Radiation Processes
}

\author{
J.S. Kaastra · F.B.S. Paerels · F. Durret • S. Schindler • \\ P. Richter
}

Received: 11 October 2007 / Accepted: 7 November 2007 / Published online: 13 February 2008

(C) Springer Science+Business Media B.V. 2008

\begin{abstract}
We discuss the different physical processes that are important to understand the thermal X-ray emission and absorption spectra of the diffuse gas in clusters of galaxies and the warm-hot intergalactic medium. The ionisation balance, line and continuum emission and absorption properties are reviewed and several practical examples are given that illustrate the most important diagnostic features in the X-ray spectra.
\end{abstract}

Keywords Atomic processes - Radiation mechanisms: thermal · Intergalactic medium · $\mathrm{X}$-rays: general

\section{Introduction}

Thermal X-ray radiation is an important diagnostic tool for studying cosmic sources where high-energy processes are important. Examples are the hot corona of the Sun and of stars,

J.S. Kaastra $(\bowtie) \cdot$ F.B.S. Paerels

SRON Netherlands Institute for Space Research, Sorbonnelaan 2, 3584 CA Utrecht, The Netherlands e-mail: j.kaastra@sron.nl

J.S. Kaastra

Astronomical Institute, Utrecht University, P.O. Box 80000, 3508 TA Utrecht, The Netherlands

\section{F.B.S. Paerels}

Department of Astronomy, Columbia University, New York, NY, USA

F. Durret

Institut d'Astrophysique de Paris, CNRS, UMR 7095, Université Pierre et Marie Curie, 98bis Bd Arago, 75014 Paris, France

\section{S. Schindler}

Institute for Astro- and Particle Physics, University of Innsbruck, Technikerstr. 25, 6020 Innsbruck, Austria

P. Richter

Physics Institute, Potsdam University, Am Neuen Palais 10, 14469 Potsdam, Germany 
Solar and stellar flares, supernova remnants, cataclysmic variables, accretion disks in binary stars and around black holes (Galactic and extragalactic), the diffuse interstellar medium of our Galaxy or external galaxies, the outer parts of Active Galactic Nuclei (AGN), the hot intracluster medium, the diffuse intercluster medium. In all these cases there is thermal $\mathrm{X}$-ray emission or absorption.

In the present paper we focus upon the properties of the X-ray spectrum in the diffuse gas within and between galaxies, clusters and the large scale structures of the Universe. As this gas has very low densities, the level populations in the atoms are not governed by Saha-like equations, but instead most of the atoms will be in or near the ground state. This simplifies the problem considerably. Furthermore, in these tenuous media we need to consider only gas with small or moderate optical depth; the radiative transport is therefore simple. Stellar coronae have similar physical conditions, but there the densities are higher so that in several cases the emergent spectrum has density-dependent features. Due to the low densities in our sources, these density effects can be ignored in most cases; however, for the lowest density gas photoionisation effects must be taken into account.

We show in this paper that it is possible to derive many different physical parameters from an X-ray spectrum: temperature, density, chemical abundances, plasma age, degree of ionisation, irradiating continuum, geometry etc.

The outline of this paper is as follows. First, we give a brief overview of atomic structure (Sect. 2). We then discuss a few basic processes that play an important role for the thermal plasmas considered here (Sect. 3). For the proper calculation of an X-ray spectrum, three different steps should be considered:

1. the determination of the ionisation balance (Sect. 4),

2. the determination of the emitted spectrum (Sect. 5),

3. possible absorption of the photons on their way towards Earth (Sect. 6).

We then briefly discuss issues like the Galactic foreground emission (Sect. 7), plasma cooling (Sect. 8), the role of non-thermal electrons (Sect. 9), and conclude with a section on plasma modelling (Sect. 10).

\section{A Short Introduction to Atomic Structure}

\subsection{The Bohr Atom}

The electrons in an atom have orbits with discrete energy levels and quantum numbers. The principal quantum number $n$ corresponds to the energy $I_{n}$ of the orbit (in the classical Bohr model for the hydrogen atom the energy $I_{n}=E_{\mathrm{H}} n^{-2}$ with $E_{\mathrm{H}}=13.6 \mathrm{eV}$ the Rydberg energy), and it takes discrete values $n=1,2,3, \ldots$ An atomic shell consists of all electrons with the same value of $n$.

The second quantum number $\ell$ corresponds to the angular momentum of the electron, and takes discrete values $\ell<n$. Orbits with $\ell=0,1,2,3$ are designated as $\mathrm{s}, \mathrm{p}, \mathrm{d}$, and $\mathrm{f}$ orbits. A subshell consists of all electrons with the same value of $n$ and $\ell$; they are usually designated as $1 \mathrm{~s}, 2 \mathrm{~s}, 2 \mathrm{p}$, etc.

The spin quantum number $s$ of an electron can take values $s= \pm 1 / 2$, and the combined total angular momentum $j$ has a quantum number with values between $\ell-1 / 2$ (for $\ell>0$ ) and $\ell+1 / 2$. Subshells are subdivided according to their $j$-value and are designated as $n \ell_{j}$. Example: $n=2, \ell=1, j=3 / 2$ is designated as $2 \mathrm{p}_{3 / 2}$.

There is also another notation that is commonly used in X-ray spectroscopy. Shells with $n=1,2,3,4,5,6$ and 7 are indicated with $\mathrm{K}, \mathrm{L}, \mathrm{M}, \mathrm{N}, \mathrm{O}, \mathrm{P}, \mathrm{Q}$. A further subdivision is 
made starting from low values of $\ell$ up to higher values of $\ell$ and from low values of $j$ up to higher values of $j$ :

$\begin{array}{ccccccccccr}1 \mathrm{~s} & 2 \mathrm{~s} & 2 \mathrm{p}_{1 / 2} & 2 \mathrm{p}_{3 / 2} & 3 \mathrm{~s} & 3 \mathrm{p}_{1 / 2} & 3 \mathrm{p}_{3 / 2} & 3 \mathrm{~d}_{3 / 2} & 3 \mathrm{~d}_{5 / 2} & 4 \mathrm{~s} & \text { etc. } \\ \mathrm{K} & \mathrm{L}_{I} & \mathrm{~L}_{I I} & \mathrm{~L}_{I I I} & \mathrm{M}_{I} & \mathrm{M}_{I I} & \mathrm{M}_{I I I} & \mathrm{M}_{I V} & \mathrm{M}_{V} & \mathrm{~N}_{I} & \\ 2 & 2 & 2 & 4 & 2 & 2 & 4 & 4 & 6 & 2 & \end{array}$

The third row in this table indicates the maximum number of electrons that can be contained in each subshell. This so-called statistical weight is simply $2 j+1$.

Atoms or ions with multiple electrons in most cases have their shells filled starting from low to high $n$ and $\ell$. For example, neutral oxygen has 8 electrons, and the shells are filled like $1 s^{2} 2 s^{2} 2 p^{4}$, where the superscripts denote the number of electrons in each shell. Ions or atoms with completely filled subshells (combining all allowed $j$-values) are particularly stable. Examples are the noble gases: neutral helium, neon and argon, and more general all ions with 2,10 or 18 electrons. In chemistry, it is common practise to designate ions by the number of electrons that they have lost, like $\mathrm{O}^{2+}$ for doubly ionised oxygen. In astronomical spectroscopic practise, more often one starts to count with the neutral atom, such that $\mathrm{O}^{2+}$ is designated as O III. As the atomic structure and the possible transitions of an ion depend primarily on the number of electrons it has, there are all kinds of scaling relationships along so-called iso-electronic sequences. All ions on an iso-electronic sequence have the same number of electrons; they differ only by the nuclear charge $Z$. Along such a sequence, energies, transitions or rates are often continuous functions of $Z$. A well known example is the famous $1 s-2 p$ triplet of lines in the helium iso-electronic sequence (2-electron systems), e.g. C V, N VI and O VII.

\subsection{Level Notation in Multi-Electron Systems}

For ions or atoms with more than one electron, the atomic structure is determined by the combined quantum numbers of the electrons. These quantum numbers have to be added according to the rules of quantum mechanics. We will not go into detail here, but refer to textbooks such as Herzberg (1944). The determination of the allowed quantum states and the transitions between these states can be rather complicated. Important here is to know that for each electron configuration (for example 1s $2 p$ ) there is a number of allowed terms or multiplets, designated as ${ }^{2 S+1} L$, with $S$ the combined electron spin (derived from the individual $s$ values of the electrons), and $L$ represents the combined angular momentum (from the individual $\ell$ values). For $L$ one usually substitutes the alphabetic designations similar to those for single electrons, namely $\mathrm{S}$ for $L=0, \mathrm{P}$ for $L=1$, etc. The quantity $2 S+1$ represents the multiplicity of the term, and gives the number of distinct energy levels of the term. The energy levels of each term can be distinguished by $J$, the combined total angular momentum quantum number $j$ of the electrons. Terms with $2 S+1$ equals 1,2 or 3 are designated as singlets, doublets and triplets, etc.

For example, a configuration with two equivalent p electrons (e.g., $3 \mathrm{p}^{2}$ ), has three allowed terms, namely ${ }^{1} \mathrm{~S},{ }^{1} \mathrm{D}$ and ${ }^{3} \mathrm{P}$. The triplet term ${ }^{3} \mathrm{P}$ has $2 S+1=3$ hence $S=1$ and $L=1$ (corresponding to $\mathrm{P}$ ), and the energy levels of this triplet are designated as ${ }^{3} \mathrm{P}_{0},{ }^{3} \mathrm{P}_{1}$ and ${ }^{3} \mathrm{P}_{2}$ according to their $J$ values 0,1 and 2 .

\subsection{Binding Energies}

The binding energy $I$ of K-shell electrons in neutral atoms increases approximately as $I \sim Z^{2}$ with $Z$ being the nuclear charge (see Table 1 and Fig. 1). Also for other shells the energy increases strongly with increasing nuclear charge $Z$. 
Fig. 1 Energy levels of atomic subshells for neutral atoms

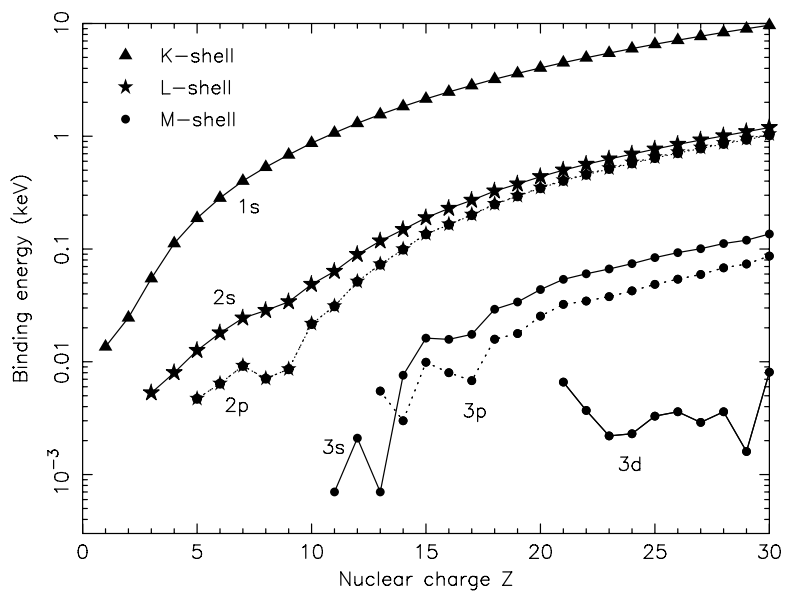

Table 1 Binding energies $E$, corresponding wavelengths $\lambda$ and photoionisation cross sections $\sigma$ at the edges of neutral atoms (nuclear charge $Z$ ) for abundant elements. Only edges in the X-ray band are given. Note that 1 Mbarn $=10^{-22} \mathrm{~m}^{2}$

\begin{tabular}{|c|c|c|c|c|c|c|c|c|c|}
\hline & $Z$ & $\begin{array}{l}E \\
(\mathrm{eV})\end{array}$ & $\begin{array}{l}\lambda \\
(\AA)\end{array}$ & $\begin{array}{l}\sigma \\
\text { (Mbarn) }\end{array}$ & & $Z$ & $\begin{array}{l}E \\
(\mathrm{eV})\end{array}$ & $\begin{array}{l}\lambda \\
(\AA)\end{array}$ & $\begin{array}{l}\sigma \\
\text { (Mbarn) }\end{array}$ \\
\hline \multicolumn{5}{|c|}{ 1s shell: } & \multicolumn{5}{|c|}{ 2s shell: } \\
\hline $\mathrm{H}$ & 1 & 13.6 & 911.8 & 6.29 & $\mathrm{O}$ & 8 & 16.6 & 747.3 & 1.37 \\
\hline $\mathrm{He}$ & 2 & 24.6 & 504.3 & 7.58 & $\mathrm{Si}$ & 14 & 154 & 80.51 & 0.48 \\
\hline $\mathrm{C}$ & 6 & 288 & 43.05 & 0.96 & S & 16 & 232 & 53.44 & 0.37 \\
\hline $\mathrm{N}$ & 7 & 403 & 30.77 & 0.67 & $\mathrm{Ar}$ & 18 & 327 & 37.97 & 0.29 \\
\hline $\mathrm{O}$ & 8 & 538 & 23.05 & 0.50 & $\mathrm{Ca}$ & 20 & 441 & 28.11 & 0.25 \\
\hline $\mathrm{Ne}$ & 10 & 870 & 14.25 & 0.29 & $\mathrm{Fe}$ & 26 & 851 & 14.57 & 0.15 \\
\hline $\mathrm{Mg}$ & 12 & 1308 & 9.48 & 0.20 & $\mathrm{Ni}$ & 28 & 1015 & 12.22 & 0.13 \\
\hline $\mathrm{Si}$ & 14 & 1844 & 6.72 & 0.14 & \multicolumn{5}{|c|}{$2 \mathrm{p}_{1 / 2}$ shell: } \\
\hline S & 16 & 2476 & 5.01 & 0.096 & $S$ & 16 & 169 & 73.26 & 1.61 \\
\hline $\mathrm{Ar}$ & 18 & 3206 & 3.87 & 0.070 & $\mathrm{Ar}$ & 18 & 251 & 49.46 & 1.45 \\
\hline $\mathrm{Ca}$ & 20 & 4041 & 3.07 & 0.060 & $\mathrm{Ca}$ & 20 & 353 & 35.16 & 0.86 \\
\hline $\mathrm{Fe}$ & 26 & 7117 & 1.74 & 0.034 & $\mathrm{Fe}$ & 26 & 726 & 17.08 & 0.42 \\
\hline \multirow[t]{7}{*}{$\mathrm{Ni}$} & 28 & 8338 & 1.49 & 0.029 & $\mathrm{Ni}$ & 28 & 877 & 14.13 & 0.35 \\
\hline & & & & & \multicolumn{5}{|c|}{$2 \mathrm{p}_{3 / 2}$ shell: } \\
\hline & & & & & S & 16 & 168 & 73.80 & 3.24 \\
\hline & & & & & $\mathrm{Ar}$ & 18 & 249 & 49.87 & 2.97 \\
\hline & & & & & $\mathrm{Ca}$ & 20 & 349 & 35.53 & 1.74 \\
\hline & & & & & $\mathrm{Fe}$ & 26 & 713 & 17.39 & 0.86 \\
\hline & & & & & $\mathrm{Ni}$ & 28 & 860 & 14.42 & 0.73 \\
\hline
\end{tabular}

For ions of a given element the ionisation energies decrease with decreasing ionisation stage: for lowly ionised ions, a part of the Coulomb force exerted on an electron by the positively charged nucleus is compensated by the other electrons in the ion, thereby allowing a wider orbit with less energy. An example is given in Table 2. 
Table 2 Binding energies $E$, corresponding wavelengths $\lambda$ and photoionisation cross sections $\sigma$ at the $\mathrm{K}$-edges of oxygen ions

\begin{tabular}{llll}
\hline Ion & $\begin{array}{l}E \\
(\mathrm{eV})\end{array}$ & $\begin{array}{l}\lambda \\
(\AA)\end{array}$ & $\begin{array}{l}\sigma \\
(\text { Mbarn }) \\
\left(10^{-22} \mathrm{~m}^{2}\right)\end{array}$ \\
\hline O I & 544 & 22.77 & 0.50 \\
O II & 565 & 21.94 & 0.45 \\
O III & 592 & 20.94 & 0.41 \\
O IV & 618 & 20.06 & 0.38 \\
O V & 645 & 19.22 & 0.35 \\
O VI & 671 & 18.48 & 0.32 \\
O VII & 739 & 16.77 & 0.24 \\
O VIII & 871 & 14.23 & 0.10 \\
\hline & & &
\end{tabular}

Table 3 Proto-solar abundances for the 15 most common chemical elements. Abundances $A$ are given with respect to hydrogen. Data from Lodders (2003)

\begin{tabular}{lllclc}
\hline Element & Abundance & Element & Abundance & Element & Abundance \\
\hline $\mathrm{H}$ & $\equiv 1$ & $\mathrm{Ne}$ & $89.1 \times 10^{-6}$ & $\mathrm{~S}$ & $18.2 \times 10^{-6}$ \\
$\mathrm{He}$ & 0.0954 & $\mathrm{Na}$ & $2.34 \times 10^{-6}$ & $\mathrm{Ar}$ & $4.17 \times 10^{-6}$ \\
$\mathrm{C}$ & $288 \times 10^{-6}$ & $\mathrm{Mg}$ & $41.7 \times 10^{-6}$ & $\mathrm{Ca}$ & $2.57 \times 10^{-6}$ \\
$\mathrm{~N}$ & $79.4 \times 10^{-6}$ & $\mathrm{Al}$ & $3.47 \times 10^{-6}$ & $\mathrm{Fe}$ & $34.7 \times 10^{-6}$ \\
$\mathrm{O}$ & $575 \times 10^{-6}$ & $\mathrm{Si}$ & $40.7 \times 10^{-6}$ & $\mathrm{Ni}$ & $1.95 \times 10^{-6}$ \\
\hline
\end{tabular}

\subsection{Abundances}

With high spectral resolution and sensitivity, optical spectra of stars sometimes show spectral features from almost all elements of the Periodic Table, but in practise only a few of the most abundant elements show up in X-ray spectra of cosmic plasmas. In several situations the abundances of the chemical elements in an X-ray source are similar to (but not necessarily equal to) the abundances for the Sun or a constant fraction of that. There have been many significant changes over the last several years in the adopted set of standard cosmic abundances. A still often used set of abundances is that of Anders and Grevesse (1989), but a more recent one is the set of proto-solar abundances of Lodders (2003), that we list in Table 3 for a few of the key elements.

In general, for absorption studies the strength of the lines is mainly determined by atomic parameters that do not vary much along an iso-electronic sequence, and the abundance of the element. Therefore, in the X-ray band the oxygen lines are the strongest absorption lines, as oxygen is the most abundant metal. The emissivity of ions in the X-ray band often increases with a strong power of the nuclear charge. For that reason, in many X-ray plasmas the strongest iron lines are often of similar strength to the strongest oxygen lines, despite the fact that the cosmic abundance of iron is only $6 \%$ of the cosmic oxygen abundance. 


\section{Basic Processes}

\subsection{Excitation Processes}

A bound electron in an ion can be brought into a higher, excited energy level through a collision with a free electron or by absorption of a photon. The latter will be discussed in more detail in Sect. 6. Here we focus upon excitation by electrons.

The cross section $Q_{i j}$ for excitation from level $i$ to level $j$ for this process can be conveniently parametrised by

$$
Q_{i j}(U)=\frac{\pi a_{0}^{2}}{w_{i}} \frac{E_{\mathrm{H}}}{E_{i j}} \frac{\Omega(U)}{U},
$$

where $U=E_{i j} / E$ with $E_{i j}$ the excitation energy from level $i$ to $j, E$ the energy of the exciting electron, $E_{\mathrm{H}}$ the Rydberg energy $(13.6 \mathrm{eV}), a_{0}$ the Bohr radius and $w_{i}$ the statistical weight of the lower level $i$. The dimensionless quantity $\Omega(U)$ is the so-called collision strength. For a given transition on an iso-electronic sequence, $\Omega(U)$ is not a strong function of the atomic number $Z$, or may be even almost independent of $Z$.

Mewe (1972) introduced a convenient formula that can be used to describe most collision strengths, written here as follows:

$$
\Omega(U)=A+\frac{B}{U}+\frac{C}{U^{2}}+\frac{2 D}{U^{3}}+F \ln U,
$$

where $A, B, C, D$ and $F$ are parameters that differ for each transition. The expression can be integrated analytically over a Maxwellian electron distribution, and the result can be expressed in terms of exponential integrals. We only mention here that the total excitation rate $S_{i j}$ (in units of $\mathrm{m}^{-3} \mathrm{~s}^{-1}$ ) is given by

$$
S_{i j}=8.62 \times 10^{-12} \frac{\bar{\Omega}(y) T^{-1 / 2} \mathrm{e}^{-y}}{w_{i}},
$$

with $y \equiv E_{i j} / \mathrm{k} T$ and $\bar{\Omega}(y)$ is the Maxwellian-averaged collision strength. For low temperatures, $y \gg 1$ and $\bar{\Omega}(y)=A+B+C+2 D$, leading to $S_{i j} \sim T^{-1 / 2} \mathrm{e}^{-y}$. The excitation rate drops exponentially due to the lack of electrons with sufficient energy. In the other limit of high temperature, $y \ll 1$ and $\bar{\Omega}(y)=-F \ln y$ and hence $S_{i j} \sim T^{-1 / 2} \ln y$.

Not all transitions have this asymptotic behaviour, however. For instance, so-called forbidden transitions have $F=0$ and hence have much lower excitation rates at high energy. So-called spin-forbidden transitions even have $A=B=F=0$.

In most cases, the excited state is stable and the ion will decay back to the ground level by means of a radiative transition, either directly or through one or more steps via intermediate energy levels. Only in cases of high density or high radiation fields, collisional excitation or further radiative excitation to a higher level may become important, but for typical cluster and ISM conditions these processes are not important in most cases. Therefore, the excitation rate immediately gives the total emission line power.

\subsection{Ionisation Processes}

\subsubsection{Collisional Ionisation}

Collisional ionisation occurs when during the interaction of a free electron with an atom or ion the free electron transfers a part of its energy to one of the bound electrons, which is 
then able to escape from the ion. A necessary condition is that the kinetic energy $E$ of the free electron must be larger than the binding energy $I$ of the atomic shell from which the bound electron escapes. A formula that gives a correct order of magnitude estimate of the cross section $\sigma$ of this process and that has the proper asymptotic behaviour (first calculated by Bethe and Born) is the formula of Lotz (1968):

$$
\sigma=\frac{a n_{s} \ln (E / I)}{E I}
$$

where $n_{s}$ is the number of electrons in the shell and the normalisation $a=4.5 \times$ $10^{-24} \mathrm{~m}^{2} \mathrm{keV}^{2}$. This equation shows that high-energy electrons have less ionising power than low-energy electrons. Also, the cross section at the threshold $E=I$ is zero.

The above cross section has to be averaged over the electron distribution (Maxwellian for a thermal plasma). For simple formulae for the cross section such as (4) the integration can be done analytically and the result can be expressed in terms of exponential integrals. We give here only the asymptotic results for $C_{\mathrm{DI}}$, the total number of direct ionisations per unit volume per unit time:

$$
\mathrm{k} T \ll I: \quad C_{\mathrm{DI}} \simeq\left(\frac{2 \sqrt{2} a n_{s}}{\sqrt{\pi m_{\mathrm{e}}}}\right) \frac{n_{\mathrm{e}} n_{\mathrm{i}} \sqrt{\mathrm{k} T} \mathrm{e}^{-I / \mathrm{k} T}}{I^{2}},
$$

and

$$
\mathrm{k} T \gg I: \quad C_{\mathrm{DI}} \simeq\left(\frac{2 \sqrt{2} a n_{s}}{\sqrt{\pi m_{\mathrm{e}}}}\right) \frac{n_{\mathrm{e}} n_{\mathrm{i}} \ln (\mathrm{k} T / I)}{I \sqrt{\mathrm{k} T}} .
$$

For low temperatures, the ionisation rate therefore goes exponentially to zero. This can be understood simply, because for low temperatures only the electrons from the exponential tail of the Maxwell distribution have sufficient energy to ionise the atom or ion. For higher temperatures the ionisation rate also approaches zero, but this time because the cross section at high energies is small.

For each ion the direct ionisation rate per atomic shell can now be determined. The total rate follows immediately by adding the contributions from the different shells. Usually only the outermost two or three shells are important. That is because of the scaling with $I^{-2}$ and $I^{-1}$ in (5) and (6), respectively.

\subsubsection{Photoionisation}

This process is very similar to collisional ionisation. The difference is that in the case of photoionisation a photon instead of an electron is causing the ionisation. Further, the effective cross section differs from that for collisional ionisation. As an example Fig. 2 shows the cross section for neutral iron and Na-like iron. For the more highly ionised iron the socalled "edges" (corresponding to the ionisation potentials $I$ ) occur at higher energies than for neutral iron. Contrary to the case of collisional ionisation, the cross section at threshold for photoionisation is not zero. The effective cross section just above the edges sometimes changes rapidly (the so-called Cooper minima and maxima).

Contrary to collisional ionisation, all the inner shells now have the largest cross section. For the K-shell one can approximate for $E>I$

$$
\sigma(E) \simeq \sigma_{0}(I / E)^{3}
$$



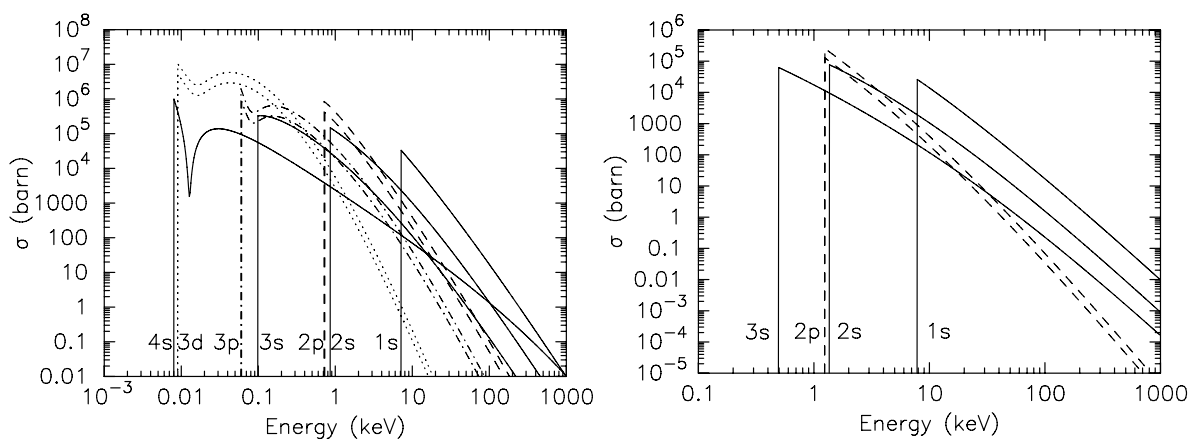

Fig. 2 Photoionisation cross section in barn $\left(10^{-28} \mathrm{~m}^{-2}\right.$ ) for Fe I (left) and Fe XVI (right). The p and $\mathrm{d}$ states (dashed and dotted) have two lines each because of splitting into two sublevels with different $j$ (see Sect. 2.1)

For a given ionising spectrum $F(E)$ (photons per unit volume per unit energy) the total number of photoionisations follows as

$$
C_{\mathrm{PI}}=c \int_{0}^{\infty} n_{\mathrm{i}} \sigma(E) F(E) \mathrm{d} E .
$$

For hydrogenlike ions one can write:

$$
\sigma_{\mathrm{PI}}=\frac{64 \pi n g(E, n) \alpha a_{0}^{2}}{3 \sqrt{3} Z^{2}}\left(\frac{I}{E}\right)^{3},
$$

where $n$ is the principal quantum number, $\alpha$ the fine structure constant and $a_{0}$ the Bohr radius. The Gaunt factor $g(E, n)$ is of order unity and varies only slowly as a function of $E$. It has been calculated and tabulated by Karzas and Latter (1961). The above equation is also applicable to excited states of the atom, and is a good approximation for all excited atoms or ions where $n$ is larger than the corresponding value for the valence electron.

\subsubsection{Compton Ionisation}

Scattering of a photon on an electron generally leads to energy transfer from one of the particles to the other. In most cases only scattering on free electrons is considered. But Compton scattering also can occur on bound electrons. If the energy transfer from the photon to the electron is large enough, the ionisation potential can be overcome leading to ionisation. This is the Compton ionisation process.

In the Thomson limit the differential cross section for Compton scattering is given by

$$
\frac{\mathrm{d} \sigma}{\mathrm{d} \Omega}=\frac{3 \sigma_{\mathrm{T}}}{16 \pi}\left(1+\cos ^{2} \theta\right),
$$

with $\theta$ the scattering angle and $\sigma_{\mathrm{T}}$ the Thomson cross section $\left(6.65 \times 10^{-29} \mathrm{~m}^{-2}\right)$. The energy transfer $\Delta E$ during the scattering is given by ( $E$ is the photon energy):

$$
\Delta E=\frac{E^{2}(1-\cos \theta)}{m_{\mathrm{e}} \mathrm{c}^{2}+E(1-\cos \theta)} .
$$


Fig. 3 Compton ionisation cross section for neutral atoms of $\mathrm{H}$, $\mathrm{He}, \mathrm{C}, \mathrm{N}, \mathrm{O}, \mathrm{Ne}$ and $\mathrm{Fe}$

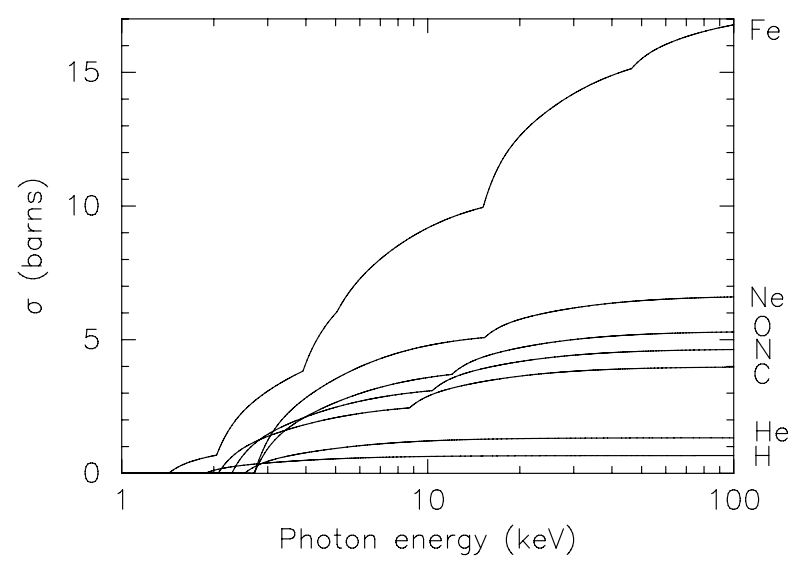

Only those scatterings where $\Delta E>I$ contribute to the ionisation. This defines a critical angle $\theta_{\mathrm{c}}$, given by:

$$
\cos \theta_{\mathrm{c}}=1-\frac{m_{\mathrm{e}} \mathrm{c}^{2} I}{E^{2}-I E} .
$$

For $E \gg I$ we have $\sigma(E) \rightarrow \sigma_{\mathrm{T}}$ (all scatterings lead in that case to ionisation) and further for $\theta_{\mathrm{c}} \rightarrow \pi$ we have $\sigma(E) \rightarrow 0$. Because for most ions $I \ll m_{\mathrm{e}} \mathrm{c}^{2}$, this last condition occurs for $E \simeq \sqrt{I m_{\mathrm{e}} \mathrm{c}^{2} / 2} \gg I$. See Fig. 3 for an example of some cross sections. In general, Compton ionisation is important if the ionising spectrum contains a significant hard X-ray contribution, for which the Compton cross section is larger than the steeply falling photoionisation cross section.

\subsubsection{Autoionisation and Fluorescence}

As we showed above, interaction of a photon or free electron with an atom or ion may lead to ionisation. In particular when an electron from one of the inner shells is removed, the resulting ion has a "vacancy" in its atomic structure and is unstable. Two different processes may occur to stabilise the ion again.

The first process is fluorescence. Here one of the electrons from the outer shells makes a radiative transition in order to occupy the vacancy. The emitted photon has an energy corresponding to the energy difference between the initial and final discrete states.

The other possibility to fill the gap is auto-ionisation through the Auger process. In this case, also one of the electrons from the outer shells fills the vacancy in the lower level. The released energy is not emitted as a photon, however, but transferred to another electron from the outer shells that is therefore able to escape from the ion. As a result, the initial ionisation may lead to a double ionisation. If the final electron configuration of the ion still has holes, more auto-ionisations or fluorescence may follow until the ion has stabilised.

In Fig. 4 the fluorescence yield $\omega$ (probability that a vacancy will be filled by a radiative transition) is shown for all elements. In general, the fluorescence yield increases strongly with increasing nuclear charge $Z$, and is higher for the innermost atomic shells. As a typical example, for Fe I a K-shell vacancy has $\omega=0.34$, while an $\mathrm{L}_{I}$-shell vacancy has $\omega=0.001$. For O I these numbers are 0.009 and 0 , respectively. 

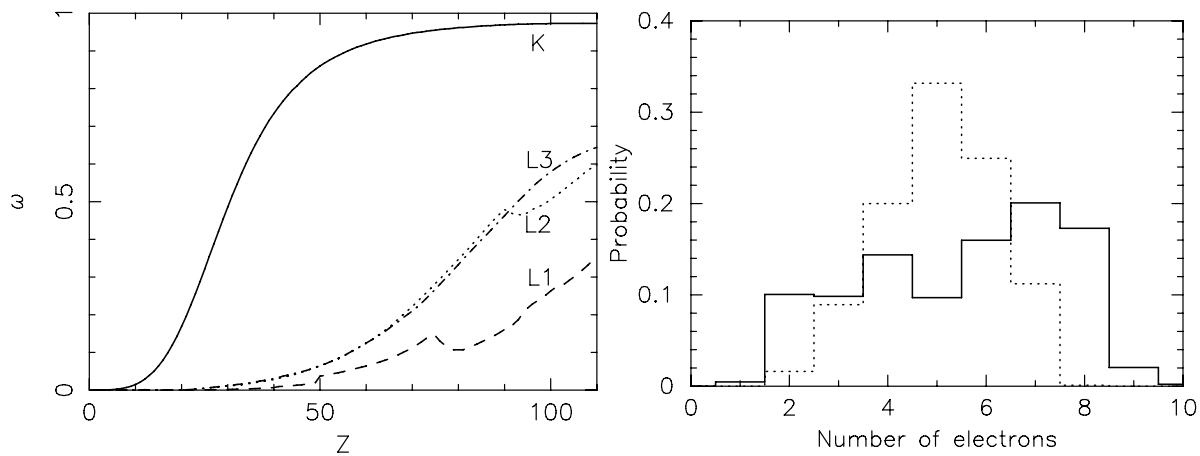

Fig. 4 Left panel: Fluorescence yield $\omega$ as a function of atomic number $Z$ for the $\mathrm{K}$ and $\mathrm{L}$ shells. Right panel: Distribution of number of electrons liberated after the initial removal of an electron from the K-shell (solid line) or $\mathrm{L}_{I}$ shell (dotted line), including the original photo-electron, for Fe I; after Kaastra and Mewe (1993)

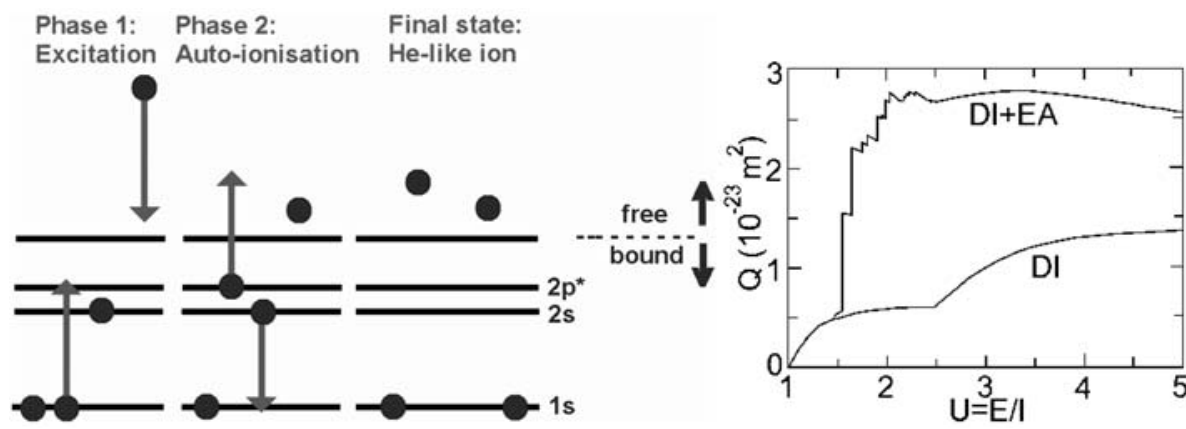

Fig. 5 Left panel: the Excitation-Autoionisation process for a Li-like ion. Right panel: collisional ionisation cross section for Fe XVI. The contribution from direct collisional ionisation (DI) and excitation-autoionisation (EA) are indicated. Adapted from Arnaud and Rothenflug (1985)

\subsubsection{Excitation-Autoionisation}

In Sect. 3.2.1 we showed how the collision of a free electron with an ion can lead to ionisation. In principle, if the free electron has insufficient energy $(E<I)$, there will be no ionisation. However, even in that case it is sometimes still possible to ionise, but in a more complicated way. The process is as follows. The collision can bring one of the electrons in the outermost shells in a higher quantum level (excitation). In most cases, the ion will return to its ground level by a radiative transition. But in some cases the excited state is unstable, and a radiationless Auger transition can occur (see Sect. 3.2.4). The vacancy that is left behind by the excited electron is being filled by another electron from one of the outermost shells, while the excited electron is able to leave the ion (or a similar process with the role of both electrons reversed).

Because of energy conservation, this process only occurs if the excited electron comes from one of the inner shells (the ionisation potential barrier must be taken anyhow). The process is in particular important for Li-like and Na-like ions, and for several other individual atoms and ions. As an example we treat here Li-like ions (see Fig. 5, left panel). In that case the most important contribution comes from a $1 \mathrm{~s}-2 \mathrm{p}$ excitation. 


\subsection{Recombination Processes}

\subsubsection{Radiative Recombination}

Radiative recombination is the reverse process of photoionisation. A free electron is captured by an ion while emitting a photon. The released radiation is the so-called free-bound continuum emission. It is relatively easy to show that there is a simple relation between the photoionisation cross section $\sigma_{\mathrm{bf}}(E)$ and the recombination cross section $\sigma_{\mathrm{fb}}$, namely the Milne-relation:

$$
\sigma_{\mathrm{fb}}(v)=\frac{E^{2} g_{n} \sigma_{\mathrm{bf}}(E)}{m_{\mathrm{e}} c^{2} m_{\mathrm{e}} v^{2}}
$$

where $g_{n}$ is the statistical weight of the quantum level into which the electron is captured (for an empty shell this is $g_{n}=2 n^{2}$ ). By averaging over a Maxwell distribution one gets the recombination-coefficient to level $n$ :

$$
R_{n}=n_{\mathrm{i}} n_{\mathrm{e}} \int_{0}^{\infty} v f(v) \sigma_{\mathrm{fb}}(v) \mathrm{d} v .
$$

Of course there is energy conservation, so $E=\frac{1}{2} m_{\mathrm{e}} v^{2}+I$.

It can be shown that for the photoionisation cross section (9) and for $g=1$, constant and $g_{n}=2 n^{2}$ :

$$
R_{n}=\frac{128 \sqrt{2 \pi} n^{3} \alpha a_{0}^{2} I^{3} \mathrm{e}^{I / \mathrm{k} T} n_{\mathrm{i}} n_{\mathrm{e}}}{3 \sqrt{3 m_{\mathrm{e}} \mathrm{k} T} Z^{2} \mathrm{k} T m_{\mathrm{e}} \mathrm{c}^{3}} E_{1}(I / \mathrm{k} T) .
$$

With the asymptotic relations for the exponential integrals it can be shown that

$$
\begin{array}{ll}
\mathrm{k} T \ll I: & R_{n} \sim T^{-\frac{1}{2}}, \\
\mathrm{k} T \gg I: & R_{n} \sim \ln (I / \mathrm{k} T) T^{-3 / 2} .
\end{array}
$$

Therefore for $T \rightarrow 0$ the recombination coefficient approaches infinity: a cool plasma is hard to ionise. For $T \rightarrow \infty$ the recombination coefficient goes to zero, because of the Milne relation $(v \rightarrow \infty)$ and because of the sharp decrease of the photoionisation cross section for high energies.

As a rough approximation we can use further that $I \sim(Z / n)^{2}$. Substituting this we find that for $\mathrm{k} T \ll I$ (recombining plasmas) $R_{n} \sim n^{-1}$, while for $\mathrm{k} T \gg I$ (ionising plasmas) $R_{n} \sim n^{-3}$. In recombining plasmas in particular many higher excited levels will be populated by the recombination, leading to significantly stronger line emission. On the other hand, in ionising plasmas (such as supernova remnants) recombination mainly occurs to the lowest levels. Note that for recombination to the ground level the approximation (15) cannot be used (the hydrogen limit), but instead one should use the exact photoionisation cross section of the valence electron. By adding over all values of $n$ and applying an approximation Seaton (1959) found for the total radiative recombination rate $\alpha_{\mathrm{RR}}$ (in units of $\mathrm{m}^{-3} \mathrm{~s}^{-1}$ ):

$$
\alpha_{\mathrm{RR}} \equiv \sum_{n} R_{n}=5.197 \times 10^{-20} Z \lambda^{1 / 2}\left\{0.4288+0.5 \ln \lambda+0.469 \lambda^{-1 / 3}\right\}
$$

with $\lambda \equiv E_{\mathrm{H}} Z^{2} / \mathrm{k} T$ and $E_{\mathrm{H}}$ the Rydberg energy (13.6 eV). Note that this equation only holds for hydrogen-like ions. For other ions usually an analytical fit to numerical calculations is 
used:

$$
\alpha_{\mathrm{RR}} \sim T^{-\eta}
$$

where the approximation is strictly speaking only valid for $T$ near the equilibrium concentration. The approximations (16) and (17) raise suspicion that for $T \rightarrow 0$ or $T \rightarrow \infty$ (19) could be a poor choice.

The captured electron does not always reach the ground level immediately. We have seen before that in particular for cool plasmas $(\mathrm{k} T \ll I)$ the higher excited levels are frequently populated. In order to get to the ground state, one or more radiative transitions are required. Apart from cascade corrections from and to higher levels the recombination line radiation is essentially given by (15). A comparison of recombination with excitation tells that in particular for low temperatures (compared to the line energy) recombination radiation dominates, and for high temperatures excitation radiation dominates. This is also one of the main differences between photoionised and collisionally ionised plasmas, as photoionised plasmas in general have a low temperature compared to the typical ionisation potentials.

\subsubsection{Dielectronic Recombination}

This process is more or less the inverse of excitation-autoionisation. Now a free electron interacts with an ion, by which it is caught (quantum level $n^{\prime \prime} \ell^{\prime \prime}$ ) but at the same time it excites an electron from $(n \ell) \rightarrow\left(n^{\prime} \ell^{\prime}\right)$. The doubly excited state is in general not stable, and the ion will return to its original state by auto-ionisation. However there is also a possibility that one of the excited electrons (usually the electron that originally belonged to the ion) falls back by a radiative transition to the ground level, creating therefore a stable, albeit excited state $\left(n^{\prime \prime} \ell^{\prime \prime}\right)$ of the ion. In particular excitations with $\ell^{\prime}=\ell+1$ contribute much to this process. In order to calculate this process, one should take account of many combinations $\left(n^{\prime} \ell^{\prime}\right)\left(n^{\prime \prime} \ell^{\prime \prime}\right)$.

The final transition probability is often approximated by

$$
\alpha_{\mathrm{DR}}=\frac{A}{T^{3 / 2}} \mathrm{e}^{-T_{0} / T}\left(1+B \mathrm{e}^{-T_{1} / T}\right)
$$

where $A, B, T_{0}$ and $T_{1}$ are adjustable parameters. Note that for $T \rightarrow \infty$ the asymptotic behaviour is identical to the case of radiative recombination. For $T \rightarrow 0$ however, dielectronic recombination can be neglected; this is because the free electron has insufficient energy to excite a bound electron. Dielectronic recombination is a dominant process in the Solar corona, and also in other situations it is often very important.

Dielectronic recombination produces more than one line photon. Consider for example the dielectronic recombination of a He-like ion into a Li-like ion:

$$
\mathrm{e}+1 \mathrm{~s}^{2} \rightarrow 1 \mathrm{~s} 2 \mathrm{p} 3 \mathrm{~s} \rightarrow 1 \mathrm{~s}^{2} 3 \mathrm{~s}+\mathrm{h} v_{1} \rightarrow 1 \mathrm{~s}^{2} 2 \mathrm{p}+\mathrm{h} v_{2} \rightarrow 1 \mathrm{~s}^{2} 2 \mathrm{~s}+\mathrm{h} v_{3}
$$

The first arrow corresponds to the electron capture, the second arrow to the stabilising radiative transition $2 p \rightarrow 1 s$ and the third arrow to the radiative transition $3 s \rightarrow 2 p$ of the captured electron. This last transition would have also occurred if the free electron was caught directly into the $3 \mathrm{~s}$ shell by normal radiative recombination. Finally, the electron has to decay further to the ground level and this can go through the normal transitions in a Li-like ion (fourth arrow). This single recombination thus produces three line photons. 
Fig. 6 Spectrum of a plasma in collisional ionisation equilibrium with $\mathrm{k} T=2 \mathrm{keV}$, near the Fe-K complex. Lines are labelled using the most common designations in this field. The Fe XXV "triplet" consists of the resonance line (w), intercombination line (actually split into $\mathrm{x}$ and $\mathrm{y}$ ) and the forbidden line $(\mathrm{z})$. All other lines are satellite lines. The labelled satellites are lines from Fe XXIV, most of the lines with energy below the forbidden $(\mathrm{z})$ line are from Fe XXIII. The relative intensity of these satellites is a strong indicator for the physical conditions in the source

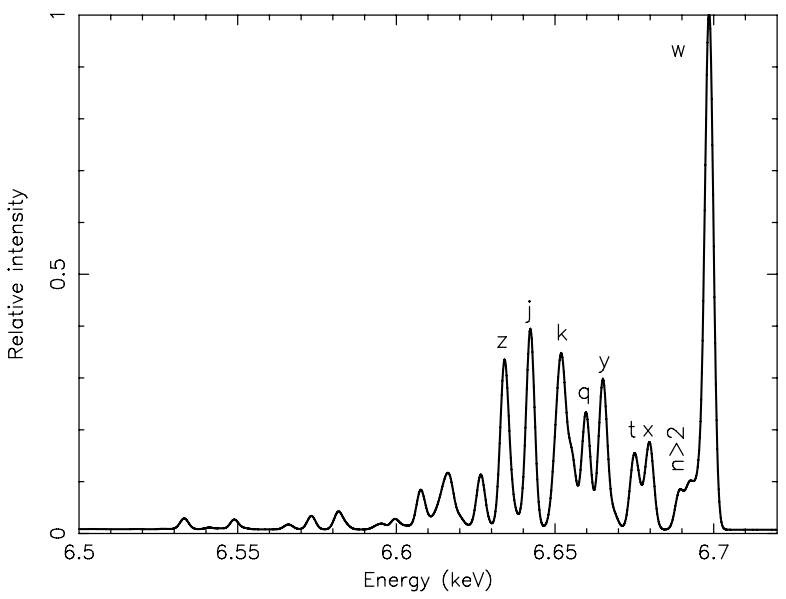

Because of the presence of the extra electron in the higher orbit, the energy $h v_{1}$ of the $2 \mathrm{p} \rightarrow 1 \mathrm{~s}$ transition is slightly different from the energy in a normal He-like ion. The stabilising transition is therefore also called a satellite line. Because there are many different possibilities for the orbit of the captured electron, one usually finds a forest of such satellite lines surrounding the normal $2 \mathrm{p} \rightarrow 1 \mathrm{~s}$ excitation line in the He-like ion (or analogously for other iso-electronic sequences). Fig. 6 gives an example of these satellite lines.

\subsection{Charge Transfer Processes}

In most cases ionisation or recombination in collisionally ionised plasmas is caused by interactions of an ion with a free electron. At low temperatures (typically below $10^{5} \mathrm{~K}$ ) also charge transfer reactions become important. During the interaction of two ions, an electron may be transferred from one ion to the other; it is usually captured in an excited state, and the excited ion is stabilised by one or more radiative transitions. As hydrogen and helium outnumber by at least a factor of 100 any other element (see Table 3), in practise only interactions between those elements and heavier nuclei are important. Reactions with $\mathrm{H} \mathrm{I}$ and $\mathrm{He}$ I lead to recombination of the heavier ion, and reactions with $\mathrm{H}$ II and $\mathrm{He}$ II to ionisation.

The electron captured during charge transfer recombination of an oxygen ion (for instance O VII, O VIII) is usually captured in an intermediate quantum state (principal quantum number $n=4-6$ ). This leads to enhanced line emission from that level as compared to the emission from other principal levels, and this signature can be used to show the presence of charge transfer reactions. Another signature - actually a signature for all recombining plasmas - is of course the enhancement of the forbidden line relative to the resonance line in the O VII triplet (Sect. 5.2.2).

An important example is the charge transfer of highly charged ions from the Solar wind with the neutral or weakly ionised Geocorona. Whenever the Sun is more active, this process may produce enhanced thermal soft X-ray emission in addition to the steady foreground emission from our own Galaxy. See Bhardwaj et al. (2006) for a review of X-rays from the Solar System. Care should be taken not to confuse this temporary enhanced Geocoronal emission with soft excess emission in a background astrophysical source. 


\section{Ionisation Balance}

In order to calculate the X-ray emission or absorption from a plasma, apart from the physical conditions also the ion concentrations must be known. These ion concentrations can be determined by solving the equations for ionisation balance (or in more complicated cases by solving the time-dependent equations). A basic ingredient in these equations are the ionisation and recombination rates, that we discussed in the previous section. Here we consider three of the most important cases: collisional ionisation equilibrium, non-equilibrium ionisation and photoionisation equilibrium.

\subsection{Collisional Ionisation Equilibrium (CIE)}

The simplest case is a plasma in collisional ionisation equilibrium (CIE). In this case one assumes that the plasma is optically thin for its own radiation, and that there is no external radiation field that affects the ionisation balance.

Photo-ionisation and Compton ionisation therefore can be neglected in the case of CIE. This means that in general each ionisation leads to one additional free electron, because the direct ionisation and excitation-autoionisation processes are most efficient for the outermost atomic shells. The relevant ionisation processes are collisional ionisation and excitationautoionisation, and the recombination processes are radiative recombination and dielectronic recombination. Apart from these processes, at low temperatures also charge transfer ionisation and recombination are important.

We define $R_{z}$ as the total recombination rate of an ion with charge $z$ to charge $z-1$, and $I_{z}$ as the total ionisation rate for charge $z$ to $z+1$. Ionisation equilibrium then implies that the net change of ion concentrations $n_{z}$ should be zero:

$$
z>0: \quad n_{z+1} R_{z+1}-n_{z} R_{z}+n_{z-1} I_{z-1}-n_{z} I_{z}=0
$$

and in particular for $z=0$ one has

$$
n_{1} R_{1}=n_{0} I_{0}
$$

(a neutral atom cannot recombine further and it cannot be created by ionisation). Next an arbitrary value for $n_{0}$ is chosen, and (23) is solved:

$$
n_{1}=n_{0}\left(I_{0} / R_{1}\right)
$$

This is substituted into (22) which now can be solved. Using induction, it follows that

$$
n_{z+1}=n_{z}\left(I_{z} / R_{z+1}\right) .
$$

Finally everything is normalised by demanding that

$$
\sum_{z=0}^{Z} n_{z}=n_{\text {element }}
$$

where $n_{\text {element }}$ is the total density of the element, determined by the total plasma density and the chemical abundances.

Examples of plasmas in CIE are the Solar corona, coronae of stars, the hot intracluster medium, the diffuse Galactic ridge component. Figure 7 shows the ion fractions as a function of temperature for two important elements. 

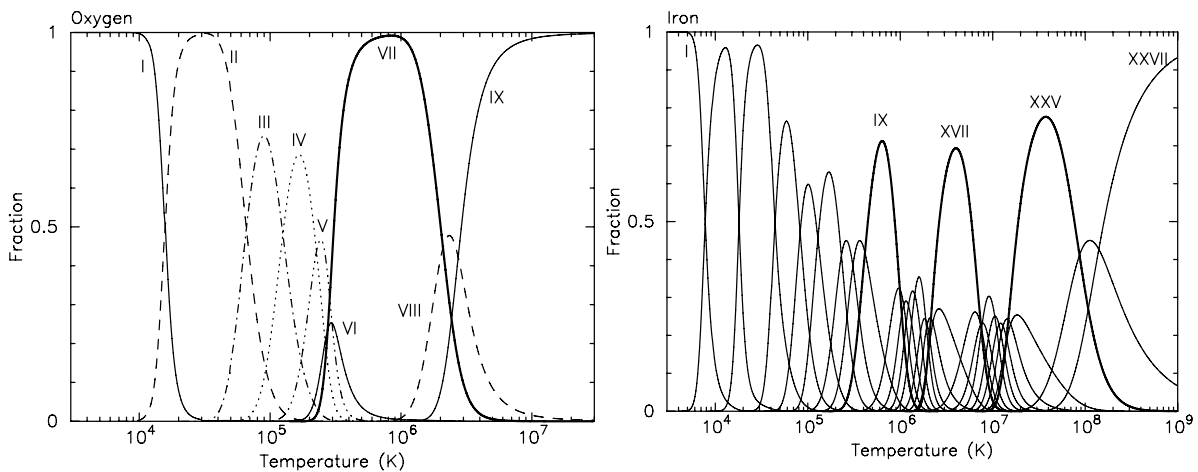

Fig. 7 Ion concentration of oxygen ions (left panel) and iron ions (right panel) as a function of temperature in a plasma in Collisional Ionisation Equilibrium (CIE). Ions with completely filled shells are indicated with thick lines: the He-like ions O VII and Fe XXV, the Ne-like Fe XVII and the Ar-like Fe IX; note that these ions are more prominent than their neighbours

\subsection{Non-Equilibrium Ionisation (NEI)}

The second case that we discuss is non-equilibrium ionisation (NEI). This situation occurs when the physical conditions of the source, like the temperature, suddenly change. A shock, for example, can lead to an almost instantaneous rise in temperature. However, it takes a finite time for the plasma to respond to the temperature change, as ionisation balance must be recovered by collisions. Similar to the CIE case we assume that photoionisation can be neglected. For each element with nuclear charge $Z$ we write:

$$
\frac{1}{n_{\mathrm{e}}(t)} \frac{\mathrm{d}}{\mathrm{d} t} \mathbf{n}(Z, t)=\mathbf{A}(Z, T(t)) \mathbf{n}(Z, t)
$$

where $\mathbf{n}$ is a vector of length $Z+1$ that contains the ion concentrations, and which is normalised according to (26). The transition matrix $\mathbf{A}$ is a $(Z+1) \times(Z+1)$ matrix given by

$$
\mathbf{A}=\left(\begin{array}{cccccc}
-I_{0} & R_{1} & 0 & 0 & \ldots & \\
I_{0} & -\left(I_{1}+R_{1}\right) & R_{2} & 0 & & \\
0 & I_{1} & \ldots & \ldots & & \\
& \vdots & \ddots & \vdots & \ldots & \\
& & \ldots & R_{Z-1} & 0 & \\
& \ldots & 0 & I_{Z-2} & -\left(I_{Z-1}+R_{Z-1}\right) & R_{Z} \\
& & \ldots & 0 & I_{Z-1} & -R_{Z}
\end{array}\right) .
$$

We can write the equation in this form because both ionisations and recombinations are caused by collisions of electrons with ions. Therefore we have the uniform scaling with $n_{\mathrm{e}}$. In general, the set of equations (27) must be solved numerically. The time evolution of the plasma can be described in general well by the parameter

$$
U=\int n_{\mathrm{e}} \mathrm{d} t
$$


The integral should be done over a co-moving mass element. Typically, for most ions equilibrium is reached for $U \sim 10^{18} \mathrm{~m}^{-3} \mathrm{~s}$. We should mention here, however, that the final outcome also depends on the temperature history $T(t)$ of the mass element, but in most cases the situation is simplified to $T(t)=$ constant.

\subsection{Photoionisation Equilibrium (PIE)}

The third case that we treat are the photoionised plasmas. Usually one assumes equilibrium (PIE), but there are of course also extensions to non-equilibrium photo-ionised situations. Apart from the same ionisation and recombination processes that play a role for plasmas in NEI and CIE, also photoionisation and Compton ionisation are relevant. Because of the multiple ionisations caused by Auger processes, the equation for the ionisation balance is not as simple as (22), because now one needs to couple more ions. Moreover, not all rates scale with the product of electron and ion density, but the balance equations also contain terms proportional to the product of ion density times photon density. In addition, apart from the equation for the ionisation balance, one needs to solve simultaneously an energy balance equation for the electrons. In this energy equation not only terms corresponding to ionisation and recombination processes play a role, but also several radiation processes (Bremsstrahlung, line radiation) or Compton scattering. The equilibrium temperature must be determined in an iterative way. A classical paper describing such photoionised plasmas is Kallman and McCray (1982).

\section{Emission Processes}

\subsection{Continuum Emission Processes}

In any plasma there are three important continuum emission processes, that we briefly mention here: Bremsstrahlung, free-bound emission and two-photon emission.

\subsubsection{Bremsstrahlung}

Bremsstrahlung is caused by a collision between a free electron and an ion. The emissivity $\epsilon_{\mathrm{ff}}\left(\right.$ photons $\mathrm{m}^{-3} \mathrm{~s}^{-1} \mathrm{~J}^{-1}$ ) can be written as:

$$
\epsilon_{\mathrm{ff}}=\frac{2 \sqrt{2} \alpha \sigma_{\mathrm{T}} c n_{\mathrm{e}} n_{\mathrm{i}} Z_{\mathrm{eff}}^{2}}{\sqrt{3 \pi} E}\left(\frac{m_{\mathrm{e}} c^{2}}{\mathrm{k} T}\right)^{\frac{1}{2}} g_{\mathrm{ff}} e^{-E / \mathrm{k} T},
$$

where $\alpha$ is the fine structure constant, $\sigma_{\mathrm{T}}$ the Thomson cross section, $n_{\mathrm{e}}$ and $n_{\mathrm{i}}$ the electron and ion density, and $E$ the energy of the emitted photon. The factor $g_{\mathrm{ff}}$ is the so-called Gaunt factor and is a dimensionless quantity of order unity. Further, $Z_{\text {eff }}$ is the effective charge of the ion, defined as

$$
Z_{\mathrm{eff}}=\left(\frac{n_{r}^{2} I_{r}}{E_{\mathrm{H}}}\right)^{\frac{1}{2}}
$$

where $E_{\mathrm{H}}$ is the ionisation energy of hydrogen $(13.6 \mathrm{eV}), I_{r}$ the ionisation potential of the ion after a recombination, and $n_{r}$ the corresponding principal quantum number. 
It is also possible to write (29) as $\epsilon_{\mathrm{ff}}=P_{\mathrm{ff}} n_{\mathrm{e}} n_{\mathrm{i}}$ with

$$
P_{\mathrm{ff}}=\frac{3.031 \times 10^{-21} Z_{\mathrm{eff}}^{2} g_{\mathrm{eff}} \mathrm{e}^{-E / \mathrm{k} T}}{E_{\mathrm{keV}} T_{\mathrm{keV}}^{1 / 2}},
$$

where in this case $P_{\mathrm{ff}}$ is in photons $\times \mathrm{m}^{3} \mathrm{~s}^{-1} \mathrm{keV}^{-1}$ and $E_{\mathrm{keV}}$ is the energy in keV. The total amount of radiation produced by this process is given by

$$
W_{\mathrm{tot}}=\frac{4.856 \times 10^{-37} \mathrm{~W} \mathrm{~m}^{3}}{\sqrt{T_{\mathrm{keV}}}} \int_{0}^{\infty} Z_{\mathrm{eff}}^{2} g_{\mathrm{ff}} \mathrm{e}^{-E / \mathrm{k} T} \mathrm{~d} E_{\mathrm{keV}} .
$$

From (29) we see immediately that the Bremsstrahlung spectrum (expressed in $\mathrm{W} \mathrm{m}^{-3}$ $\mathrm{keV}^{-1}$ ) is flat for $E \ll \mathrm{k} T$, and for $E>\mathrm{k} T$ it drops exponentially. In order to measure the temperature of a hot plasma, one needs to measure near $E \simeq \mathrm{k} T$. The Gaunt factor $g_{\mathrm{ff}}$ can be calculated analytically; there are both tables and asymptotic approximations available. In general, $g_{\text {ff }}$ depends on both $E / \mathrm{k} T$ and $\mathrm{k} T / Z_{\text {eff }}$.

For a plasma (29) needs to be summed over all ions that are present in order to calculate the total amount of Bremsstrahlung radiation. For cosmic abundances, hydrogen and helium usually yield the largest contribution. Frequently, one defines an average Gaunt factor $G_{\mathrm{ff}}$ by

$$
G_{\mathrm{ff}}=\sum_{i}\left(\frac{n_{\mathrm{i}}}{n_{\mathrm{e}}}\right) Z_{\mathrm{eff}, i}^{2} g_{\mathrm{eff}, i}
$$

\subsubsection{Free-Bound Emission}

Free-bound emission occurs during radiative recombination (Sect. 3.3.1). The energy of the emitted photon is at least the ionisation energy of the recombined ion (for recombination to the ground level) or the ionisation energy that corresponds to the excited state (for recombination to higher levels). From the recombination rate (see Sect. 3.3.1) the free-bound emission is determined immediately:

$$
\epsilon_{\mathrm{fb}}=\sum_{i} n_{\mathrm{e}} n_{\mathrm{i}} R_{r}
$$

Also here it is possible to define an effective Gaunt factor $G_{\mathrm{fb}}$. Free-bound emission is in practise often very important. For example in CIE for $\mathrm{k} T=0.1 \mathrm{keV}$, free-bound emission is the dominant continuum mechanism for $E>0.1 \mathrm{keV}$; for $\mathrm{k} T=1 \mathrm{keV}$ it dominates above $3 \mathrm{keV}$. For $\mathrm{k} T \gg 1 \mathrm{keV}$ Bremsstrahlung is always the most important mechanism, and for $\mathrm{k} T \ll 0.1 \mathrm{keV}$ free-bound emission dominates. See also Fig. 8.

Of course, under conditions of photoionisation equilibrium free-bound emission is even more important, because there are more recombinations than in the CIE case (because $T$ is lower, at comparable ionisation).

\subsubsection{Two Photon Emission}

This process is in particular important for hydrogen-like or helium-like ions. After a collision with a free electron, an electron from a bound $1 \mathrm{~s}$ shell is excited to the $2 \mathrm{~s}$ shell. The quantummechanical selection rules do not allow that the 2 s electron decays back to the $1 \mathrm{~s}$ orbit by a 

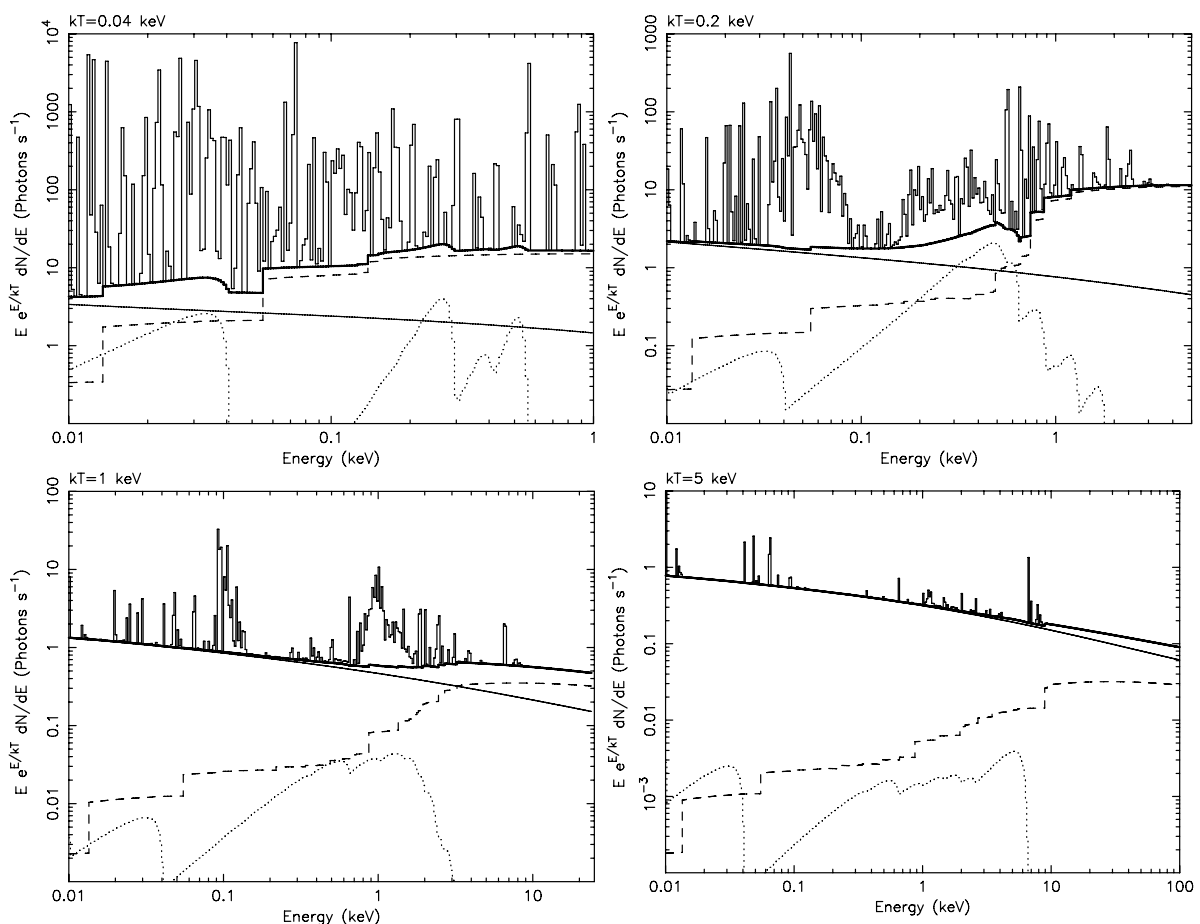

Fig. 8 Emission spectra of plasmas with solar abundances. The histogram indicates the total spectrum, including line radiation. The spectrum has been binned in order to show better the relative importance of line radiation. The thick solid line is the total continuum emission, the thin solid line the contribution due to Bremsstrahlung, the dashed line free-bound emission and the dotted line two-photon emission. Note the scaling with $E \mathrm{e}^{E / \mathrm{k} T}$ along the $y$-axis

radiative transition. Usually the ion will then be excited to a higher level by another collision, for example from $2 \mathrm{~s}$ to $2 \mathrm{p}$, and then it can decay radiatively back to the ground state (1s). However, if the density is very low $\left(n_{\mathrm{e}} \ll n_{\mathrm{e}, \text { crit }}\right.$, (36)-(37)), the probability for a second collision is very small and in that case two-photon emission can occur: the electron decays from the $2 \mathrm{~s}$ orbit to the $1 \mathrm{~s}$ orbit while emitting two photons. Energy conservation implies that the total energy of both photons should equal the energy difference between the $2 \mathrm{~s}$ and 1s level $\left(E_{2 \text { phot }}=E_{1 \mathrm{~s}}-E_{2 \mathrm{~s}}\right)$. From symmetry considerations it is clear that the spectrum must be symmetrical around $E=0.5 E_{2 \text { phot }}$, and further that it should be zero for $E=0$ and $E=E_{2 \text { phot }}$. An empirical approximation for the shape of the spectrum is given by:

$$
F(E) \sim \sqrt{\sin \left(\pi E / E_{2 \text { phot }}\right)} .
$$

An approximation for the critical density below which two photon emission is important can be obtained from a comparison of the radiative and collisional rates from the upper ( $2 \mathrm{~s})$ level, and is given by (Mewe et al. 1986):

$$
\begin{aligned}
\text { H-like : } & n_{\mathrm{e}, \text { crit }}=7 \times 10^{9} \mathrm{~m}^{-3} Z^{9.5} \\
\text { He-like : } & n_{\mathrm{e}, \text { crit }}=2 \times 10^{11} \mathrm{~m}^{-3}(Z-1)^{9.5} .
\end{aligned}
$$


For example for carbon two photon emission is important for densities below $10^{17} \mathrm{~m}^{-3}$, which is the case for many astrophysical applications. Also in this case one can determine an average Gaunt factor $G_{2 \text { phot }}$ by averaging over all ions. Two photon emission is important in practise for $0.5 \lesssim \mathrm{kT} \lesssim 5 \mathrm{keV}$, and then in particular for the contributions of $\mathrm{C}, \mathrm{N}$ and $\mathrm{O}$ between 0.2 and $0.6 \mathrm{keV}$. See also Fig. 8 .

\subsection{Line Emission Processes}

Apart from continuum radiation, line radiation plays an important role for thermal plasmas. In some cases the flux, integrated over a broad energy band, can be completely dominated by line radiation (see Fig. 8). The production process of line radiation can be broken down in two basic steps: the excitation and the emission process.

\subsubsection{Excitation Process}

An atom or ion must first be brought into an excited state before it can emit line photons. There are several physical processes that can contribute to this.

The most important process is usually collisional excitation (Sect. 3.1), in particular for plasmas in CIE. The collision of an electron with the ion brings it in an excited state.

A second way to excite the ion is by absorbing a photon with the proper energy. We discuss this process in more detail in Sect. 6.

Alternatively, inner shell ionisation (either by the collision with a free electron, Sect. 3.2.1 or by the photoelectric effect, Sect. 3.2.2) brings the ion in an excited state.

Finally, the ion can also be brought in an excited state by capturing a free electron in one of the free energy levels above the ground state (radiative recombination, Sect. 3.3.1), or through dielectronic recombination (Sect. 3.3.2).

\subsubsection{Line Emission}

It does not matter by whatever process the ion is brought into an excited state $j$, whenever it is in such a state it may decay back to the ground state or any other lower energy level $i$ by emitting a photon. The probability per unit time that this occurs is given by the spontaneous transition probability $A_{i j}$ (units: $\mathrm{s}^{-1}$ ) which is a number that is different for each transition. The total line power $P_{i j}$ (photons per unit time and volume) is then given by

$$
P_{i j}=A_{i j} n_{j}
$$

where $n_{j}$ is the number density of ions in the excited state $j$. For the most simple case of excitation from the ground state $g$ (rate $S_{g j}$ ) followed by spontaneous emission, one can simply approximate $n_{g} n_{\mathrm{e}} S_{g j}=n_{j} A_{g j}$. From this equation, the relative population $n_{j} / n_{g} \ll 1$ is determined, and then using (38) the line flux is determined. In realistic situations, however, things are more complicated. First, the excited state may also decay to other intermediate states if present, and also excitations or cascades from other levels may play a role. Furthermore, for high densities also collisional excitation or de-excitation to and from other levels becomes important. In general, one has to solve a set of equations for all energy levels of an ion where all relevant population and depopulation processes for that level are taken into account. For the resulting solution vector $n_{j}$, the emitted line power is then simply given by (38).

Note that not all possible transitions between the different energy levels are allowed. There are strict quantum mechanical selection rules that govern which lines are allowed; see 
for instance Herzberg (1944) or Mewe (1999). Sometimes there are higher order processes that still allow a forbidden transition to occur, albeit with much smaller transition probabilities $A_{i j}$. But if the excited state $j$ has no other (fast enough) way to decay, these forbidden lines occur and the lines can be quite strong, as their line power is essentially governed by the rate at which the ion is brought into its excited state $j$.

One of the most well known groups of lines is the He-like $1 \mathrm{~s}-2 \mathrm{p}$ triplet. Usually the strongest line is the resonance line, an allowed transition. The forbidden line has a similar strength as the resonance line, for the low density conditions in the ISM and intracluster medium, but it can be relatively enhanced in recombining plasmas, or relatively reduced in high density plasmas like stellar coronal loops. In between both lines is the intercombination line. In fact, this intercombination line is a doublet but for the lighter elements both components cannot be resolved. But see Fig. 6 for the case of iron.

\subsubsection{Line Width}

For most X-ray spectral lines, the line profile of a line with energy $E$ can be approximated with a Gaussian $\exp \left(-\Delta E^{2} / 2 \sigma^{2}\right)$ with $\sigma$ given by $\sigma / E=\sigma_{\mathrm{v}} / c$ where the velocity dispersion is

$$
\sigma_{\mathrm{v}}^{2}=\sigma_{\mathrm{t}}^{2}+\mathrm{k} T_{\mathrm{i}} / m_{\mathrm{i}}
$$

Here $T_{\mathrm{i}}$ is the ion temperature (not necessarily the same as the electron temperature), and $\sigma_{\mathrm{t}}$ is the root mean squared turbulent velocity of the emitting medium. For large ion temperature, turbulent velocity or high spectral resolution this line width can be measured, but in most cases the lines are not resolved for CCD type spectra.

\subsubsection{Resonance Scattering}

Resonance scattering is a process where a photon is absorbed by an atom and then re-emitted as a line photon of the same energy into a different direction. As for strong resonance lines (allowed transitions) the transition probabilities $A_{i j}$ are large, the time interval between absorption and emission is extremely short, and that is the reason why the process effectively can be regarded as a scattering process. We discuss the absorption properties in Sect. 6.3, and have already discussed the spontaneous emission in Sect. 5.2.2.

Resonance scattering of X-ray photons is potentially important in the dense cores of some clusters of galaxies for a few transitions of the most abundant elements, as first shown by Gil'fanov et al. (1987). The optical depth for scattering can be written conveniently as (cf. also Sect. 6.3):

$$
\tau=\frac{4240 f N_{24}\left(\frac{n_{\mathrm{i}}}{n_{\mathrm{Z}}}\right)\left(\frac{n_{\mathrm{Z}}}{n_{\mathrm{H}}}\right)\left(\frac{M}{T_{\mathrm{keV}}}\right)^{1 / 2}}{E_{\mathrm{keV}}\left\{1+\frac{0.0522 M v_{100}^{2}}{T_{\mathrm{keV}}}\right\}^{1 / 2}},
$$

where $f$ is the absorption oscillator strength of the line (of order unity for the strongest spectral lines), $E_{\mathrm{keV}}$ the energy in $\mathrm{keV}, N_{24}$ the hydrogen column density in units of $10^{24} \mathrm{~m}^{-2}, n_{\mathrm{i}}$ the number density of the ion, $n_{\mathrm{Z}}$ the number density of the element, $M$ the atomic weight of the ion, $T_{\mathrm{keV}}$ the ion temperature in $\mathrm{keV}$ (assumed to be equal to the electron temperature) and $v_{100}$ the micro-turbulence velocity in units of $100 \mathrm{~km} / \mathrm{s}$. Resonance scattering in clusters causes the radial intensity profile on the sky of an emission line to become weaker in the cluster core and stronger in the outskirts, without destroying photons. By comparing the radial line profiles of lines with different optical depth, for instance the $1 s-2 p$ and $1 s-3 p$ lines 
of O VII or Fe XXV, one can determine the optical depth and hence constrain the amount of turbulence in the cluster.

Another important application was proposed by Churazov et al. (2001). They show that for WHIM filaments the resonance line of the O VII triplet can be enhanced significantly compared to the thermal emission of the filament due to resonant scattering of X-ray background photons on the filament. The ratio of this resonant line to the other lines of the triplet therefore can be used to estimate the column density of a filament.

\subsection{Some Important Line Transitions}

In Tables 4 and 5 we list the 100 strongest emission lines under CIE conditions. Note that each line has its peak emissivity at a different temperature. In particular some of the H-like and He-like transitions are strong, and further the so-called Fe-L complex (lines from $n=2$ in Li-like to Ne-like iron ions) is prominent. At longer wavelengths, the L-complex of $\mathrm{Ne}$ $\mathrm{Mg}, \mathrm{Si}$ and $\mathrm{S}$ gives strong soft X-ray lines. At very short wavelengths, there are not many strong emission lines: between $6-7 \mathrm{keV}$, the Fe-K emission lines are the strongest spectral features.

\section{Absorption Processes}

\subsection{Continuum Versus Line Absorption}

$\mathrm{X}$-rays emitted by cosmic sources do not travel unattenuated to a distant observer. This is because intervening matter in the line of sight absorbs a part of the X-rays. With lowresolution instruments, absorption can be studied only through the measurement of broadband flux depressions caused by continuum absorption. However, at high spectral resolution also absorption lines can be studied, and in fact absorption lines offer more sensitive tools to detect weak intervening absorption systems. We illustrate this in Fig. 9. At an O VIII column density of $10^{21} \mathrm{~m}^{-2}$, the absorption edge has an optical depth of $1 \%$; for the same column density, the core of the Ly $\alpha$ line is already saturated and even for 100 times lower column density, the line core still has an optical depth of $5 \%$.
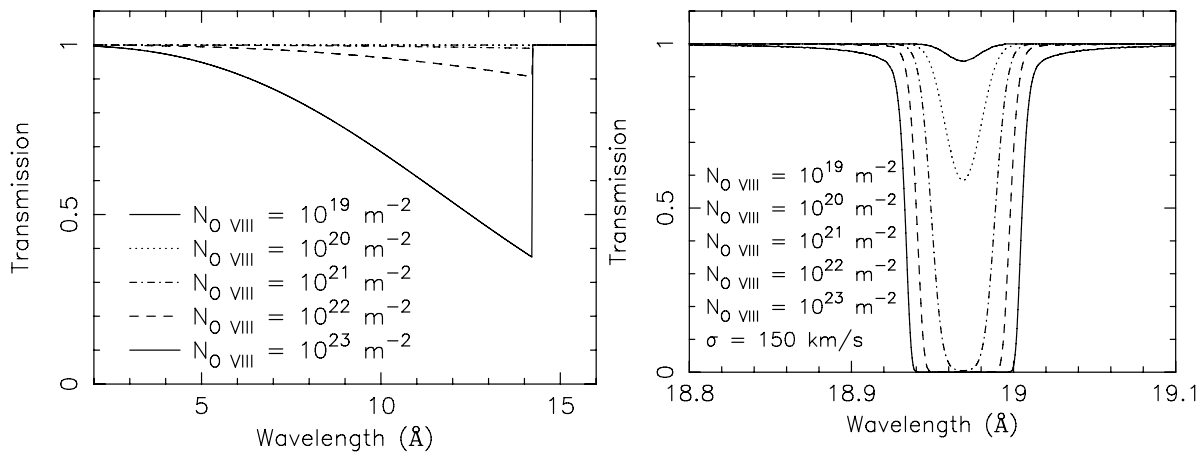

Fig. 9 Continuum (left) and Ly $\alpha$ (right) absorption spectrum for a layer consisting of O VIII ions with column densities as indicated 
Table 4 The strongest emission lines for a plasma with proto-solar abundances (Lodders 2003) in the X-ray band $43 \AA<\lambda<100 \AA$. At longer wavelengths sometimes a few lines from the same multiplet have been added. All lines include unresolved dielectronic satellites. $T_{\max }(\mathrm{K})$ is the temperature at which the emissivity peaks, $Q_{\max }=P /\left(n_{\mathrm{e}} n_{\mathrm{H}}\right)$, with $P$ the power per unit volume at $T_{\max }$, and $Q_{\max }$ is in units of $10^{-36} \mathrm{~W} \mathrm{~m}^{3}$

\begin{tabular}{|c|c|c|c|c|c|c|c|}
\hline $\begin{array}{l}E \\
(e V)\end{array}$ & $\begin{array}{l}\lambda \\
(\AA)\end{array}$ & $\begin{array}{l}-\log \\
Q_{\max }\end{array}$ & $\begin{array}{l}\log \\
T_{\max }\end{array}$ & Ion & $\begin{array}{l}\text { Iso-el. } \\
\text { seq. }\end{array}$ & $\begin{array}{l}\text { Lower } \\
\text { level }\end{array}$ & $\begin{array}{l}\text { Upper } \\
\text { level }\end{array}$ \\
\hline 126.18 & 98.260 & 1.35 & 5.82 & $\mathrm{Ne}$ VIII & $\mathrm{Li}$ & $2 \mathrm{p}^{2} \mathrm{P}_{3 / 2}$ & $3 \mathrm{~d}^{2} \mathrm{D}_{5 / 2}$ \\
\hline 126.37 & 98.115 & 1.65 & 5.82 & Ne VIII & $\mathrm{Li}$ & $2 \mathrm{p}^{2} \mathrm{P}_{1 / 2}$ & $3 \mathrm{~d}^{2} \mathrm{D}_{3 / 2}$ \\
\hline 127.16 & 97.502 & 1.29 & 5.75 & $\mathrm{Ne}$ VII & $\mathrm{Be}$ & $2 \mathrm{~s}{ }^{1} \mathrm{~S}_{0}$ & $3 \mathrm{p}^{1} \mathrm{P}_{1}$ \\
\hline 128.57 & 96.437 & 1.61 & 5.47 & Si V & $\mathrm{Ne}$ & $2 \mathrm{p}^{1} \mathrm{~S}_{0}$ & $3 \mathrm{~d}^{1} \mathrm{P}_{1}$ \\
\hline 129.85 & 95.483 & 1.14 & 5.73 & Mg VI & $\mathrm{N}$ & $2 p^{4} S_{3 / 2}$ & $3 d^{4} P_{5 / 2,3 / 2,1 / 2}$ \\
\hline 132.01 & 93.923 & 1.46 & 6.80 & Fe XVIII & $\mathrm{F}$ & $2 \mathrm{~s}^{2} \mathrm{P}_{3 / 2}$ & $2 \mathrm{p}^{2} \mathrm{~S}_{1 / 2}$ \\
\hline 140.68 & 88.130 & 1.21 & 5.75 & $\mathrm{Ne}$ VII & $\mathrm{Be}$ & $2 \mathrm{p}^{3} \mathrm{P}_{1}$ & $4 d^{3} D_{2,3}$ \\
\hline 140.74 & 88.092 & 1.40 & 5.82 & $\mathrm{Ne}$ VIII & $\mathrm{Li}$ & $2 s^{2} S_{1 / 2}$ & $3 \mathrm{p}^{2} \mathrm{P}_{3 / 2}$ \\
\hline 147.67 & 83.959 & 0.98 & 5.86 & Mg VII & $\mathrm{C}$ & $2 \mathrm{p}^{3} \mathrm{P}$ & $3 \mathrm{~d}^{3} \mathrm{D},{ }^{1} \mathrm{D},{ }^{3} \mathrm{~F}$ \\
\hline 148.01 & 83.766 & 1.77 & 5.86 & Mg VII & $\mathrm{C}$ & $2 \mathrm{p}^{3} \mathrm{P}_{2}$ & $3 \mathrm{~d}^{3} \mathrm{P}_{2}$ \\
\hline 148.56 & 83.457 & 1.41 & 5.90 & Fe IX & $\mathrm{Ar}$ & $3 \mathrm{p}^{1} \mathrm{~S}_{0}$ & $4 d^{3} P_{1}$ \\
\hline 149.15 & 83.128 & 1.69 & 5.69 & Si VI & $\mathrm{F}$ & $2 \mathrm{p}^{2} \mathrm{P}_{3 / 2}$ & $\left({ }^{3} \mathrm{P}\right) 3 \mathrm{~d}^{2} \mathrm{D}_{5 / 2}$ \\
\hline 149.38 & 83.000 & 1.48 & 5.74 & Mg VI & $\mathrm{N}$ & $2 p^{4} S_{3 / 2}$ & $4 d^{4} P_{5 / 2,3 / 2,1 / 2}$ \\
\hline 150.41 & 82.430 & 1.49 & 5.91 & Fe IX & Ar & $3 \mathrm{p}^{1} \mathrm{~S}_{0}$ & $4 \mathrm{~d}^{1} \mathrm{P}_{1}$ \\
\hline 154.02 & 80.501 & 1.75 & 5.70 & Si VI & $\mathrm{F}$ & $2 p^{2} \mathrm{P}_{3 / 2}$ & $\left({ }^{1} \mathrm{D}\right) 3 \mathrm{~d}^{2} \mathrm{D}_{5 / 2}$ \\
\hline 159.23 & 77.865 & 1.71 & 6.02 & $\mathrm{Fe} X$ & $\mathrm{Cl}$ & $3 p^{2} \mathrm{P}_{1 / 2}$ & $4 d^{2} D_{5 / 2}$ \\
\hline 165.24 & 75.034 & 1.29 & 5.94 & Mg VIII & B & $2 \mathrm{p}^{2} \mathrm{P}_{3 / 2}$ & $3 d^{2} D_{5 / 2}$ \\
\hline 165.63 & 74.854 & 1.29 & 5.94 & Mg VIII & B & $2 \mathrm{p}^{2} \mathrm{P}_{1 / 2}$ & $3 \mathrm{~d}^{2} \mathrm{D}_{3 / 2}$ \\
\hline 170.63 & 72.663 & 1.07 & 5.76 & S VII & $\mathrm{Ne}$ & $2 \mathrm{p}^{1} \mathrm{~S}_{0}$ & $3 \mathrm{~s}{ }^{3} \mathrm{P}_{1,2}$ \\
\hline 170.69 & 72.635 & 1.61 & 6.09 & $\mathrm{Fe} \mathrm{XI}$ & $\mathrm{S}$ & $3 p^{3} P_{2}$ & $4 d^{3} D_{3}$ \\
\hline 171.46 & 72.311 & 1.56 & 6.00 & Mg IX & $\mathrm{Be}$ & $2 \mathrm{p}^{1} \mathrm{P}_{1}$ & $3 \mathrm{~d}^{1} \mathrm{D}_{2}$ \\
\hline 171.80 & 72.166 & 1.68 & 6.08 & Fe XI & $\mathrm{S}$ & $3 \mathrm{p}^{1} \mathrm{D}_{2}$ & $4 \mathrm{~d}^{1} \mathrm{~F}_{3}$ \\
\hline 172.13 & 72.030 & 1.44 & 6.00 & Mg IX & $\mathrm{Be}$ & $2 \mathrm{p}^{3} \mathrm{P}_{2,1}$ & $3 s^{3} S_{1}$ \\
\hline 172.14 & 72.027 & 1.40 & 5.76 & S VII & $\mathrm{Ne}$ & $2 \mathrm{p}^{1} \mathrm{~S}_{0}$ & $3 \mathrm{~s}{ }^{1} \mathrm{P}_{1}$ \\
\hline 177.07 & 70.020 & 1.18 & 5.84 & Si VII & $\mathrm{O}$ & $2 \mathrm{p}^{3} \mathrm{P}$ & $3 \mathrm{~d}^{3} \mathrm{D},{ }^{3} \mathrm{P}$ \\
\hline 177.98 & 69.660 & 1.36 & 6.34 & Fe XV & $\mathrm{Mg}$ & $3 \mathrm{p}^{1} \mathrm{P}_{1}$ & $4 \mathrm{~s}{ }^{1} \mathrm{~S}_{0}$ \\
\hline 177.99 & 69.658 & 1.57 & 5.96 & Si VIII & $\mathrm{N}$ & $2 p^{4} S_{3 / 2}$ & $3 s{ }^{4} P_{5 / 2,3 / 2,1 / 2}$ \\
\hline 179.17 & 69.200 & 1.61 & 5.71 & Si VI & $\mathrm{F}$ & $2 p^{2} P$ & $4 \mathrm{~d}^{2} \mathrm{P},{ }^{2} \mathrm{D}$ \\
\hline 186.93 & 66.326 & 1.72 & 6.46 & Fe XVI & $\mathrm{Na}$ & $3 d^{2} D$ & $4 \mathrm{f}^{2} F$ \\
\hline 194.58 & 63.719 & 1.70 & 6.45 & Fe XVI & $\mathrm{Na}$ & $3 \mathrm{p}^{2} \mathrm{P}_{3 / 2}$ & $4 s^{2} S_{1 / 2}$ \\
\hline 195.89 & 63.294 & 1.64 & 6.08 & $\operatorname{Mg} X$ & $\mathrm{Li}$ & $2 \mathrm{p}^{2} \mathrm{P}_{3 / 2}$ & $3 \mathrm{~d}^{2} \mathrm{D}_{5 / 2}$ \\
\hline 197.57 & 62.755 & 1.46 & 6.00 & Mg IX & $\mathrm{Be}$ & $2 \mathrm{~s}{ }^{1} \mathrm{~S}_{0}$ & $3 \mathrm{p}^{1} \mathrm{P}_{1}$ \\
\hline 197.75 & 62.699 & 1.21 & 6.22 & Fe XIII & $\mathrm{Si}$ & $3 p^{3} P_{1}$ & $4 d^{3} D_{2}$ \\
\hline 198.84 & 62.354 & 1.17 & 6.22 & Fe XIII & $\mathrm{Si}$ & $3 \mathrm{p}^{3} \mathrm{P}_{0}$ & $4 d^{3} D_{1}$ \\
\hline 199.65 & 62.100 & 1.46 & 6.22 & Fe XIII & $\mathrm{Si}$ & $3 p^{3} P_{1}$ & $4 d^{3} P_{0}$ \\
\hline 200.49 & 61.841 & 1.29 & 6.07 & Si IX & $\mathrm{C}$ & $2 p^{3} P_{2}$ & $3 s^{3} P_{1}$ \\
\hline 203.09 & 61.050 & 1.06 & 5.96 & Si VIII & $\mathrm{N}$ & $2 p^{4} S_{3 / 2}$ & $3 d^{4} P_{5 / 2,3 / 2,1 / 2}$ \\
\hline 203.90 & 60.807 & 1.69 & 5.79 & S VII & $\mathrm{Ne}$ & $2 \mathrm{p}^{1} \mathrm{~S}_{0}$ & $3 \mathrm{~d}^{3} \mathrm{D}_{1}$ \\
\hline 204.56 & 60.610 & 1.30 & 5.79 & S VII & $\mathrm{Ne}$ & $2 \mathrm{p}^{1} \mathrm{~S}_{0}$ & $3 \mathrm{~d}^{1} \mathrm{P}_{1}$ \\
\hline 223.98 & 55.356 & 1.00 & 6.08 & Si IX & $\mathrm{C}$ & $2 p^{3} P$ & $3 \mathrm{~d}^{3} \mathrm{D},{ }^{1} \mathrm{D},{ }^{3} \mathrm{~F}$ \\
\hline
\end{tabular}


Table 4 (Continued)

\begin{tabular}{|c|c|c|c|c|c|c|c|}
\hline $\begin{array}{l}\mathrm{E} \\
(\mathrm{eV})\end{array}$ & $\begin{array}{l}\lambda \\
(\AA)\end{array}$ & $\begin{array}{l}-\log \\
Q_{\max }\end{array}$ & $\begin{array}{l}\log \\
T_{\max }\end{array}$ & Ion & $\begin{array}{l}\text { Iso-el. } \\
\text { seq. }\end{array}$ & $\begin{array}{l}\text { Lower } \\
\text { level }\end{array}$ & $\begin{array}{l}\text { Upper } \\
\text { level }\end{array}$ \\
\hline 234.33 & 52.911 & 1.34 & 6.34 & $\mathrm{Fe} X \mathrm{~V}$ & $\mathrm{Mg}$ & $3 \mathrm{~s}{ }^{1} \mathrm{~S}_{0}$ & $4 p^{1} \mathrm{P}_{1}$ \\
\hline 237.06 & 52.300 & 1.61 & 6.22 & Si XI & $\mathrm{Be}$ & $2 \mathrm{p}^{1} \mathrm{P}_{1}$ & $3 \mathrm{~s}^{1} \mathrm{~S}_{0}$ \\
\hline 238.43 & 52.000 & 1.44 & 5.97 & Si VIII & $\mathrm{N}$ & $2 p^{4} S_{3 / 2}$ & $4 d^{4} P_{5 / 2,3 / 2,1 / 2}$ \\
\hline 244.59 & 50.690 & 1.30 & 6.16 & SiX & B & $2 \mathrm{p}^{2} \mathrm{P}_{3 / 2}$ & $3 \mathrm{~d}^{2} \mathrm{D}_{5 / 2}$ \\
\hline 245.37 & 50.530 & 1.30 & 6.16 & $\mathrm{SiX}$ & B & $2 p^{2} \mathrm{P}_{1 / 2}$ & $3 d^{2} D_{3 / 2}$ \\
\hline 251.90 & 49.220 & 1.45 & 6.22 & Si XI & $\mathrm{Be}$ & $2 p^{1} P_{1}$ & $3 d^{1} D_{2}$ \\
\hline 252.10 & 49.180 & 1.64 & 5.97 & Ar IX & $\mathrm{Ne}$ & $2 \mathrm{p}^{1} \mathrm{~S}_{0}$ & $3 s^{3} \mathrm{P}_{1,2}$ \\
\hline 261.02 & 47.500 & 1.47 & 6.06 & S IX & $\mathrm{O}$ & $2 \mathrm{p}^{3} \mathrm{P}$ & $3 d^{3} \mathrm{D},{ }^{3} \mathrm{P}$ \\
\hline 280.73 & 44.165 & 1.60 & 6.30 & Si XII & $\mathrm{Li}$ & $2 p^{2} \mathrm{P}_{3 / 2}$ & $3 d^{2} D_{5 / 2}$ \\
\hline 283.46 & 43.740 & 1.46 & 6.22 & Si XI & $\mathrm{Be}$ & $2 \mathrm{~s}{ }^{1} \mathrm{~S}_{0}$ & $3 p{ }^{1} \mathrm{P}_{1}$ \\
\hline
\end{tabular}

\subsection{Continuum Absorption}

Continuum absorption can be calculated simply from the photoionisation cross sections, that we discussed already in Sect. 3.2.2. The total continuum opacity $\tau_{\text {cont }}$ can be written as

$$
\tau_{\text {cont }}(E) \equiv N_{\mathrm{H}} \sigma_{\text {cont }}(E)=\sum_{i} N_{i} \sigma_{i}(E)
$$

i.e. by averaging over the various ions $i$ with column density $N_{i}$. Accordingly, the continuum transmission $T(E)$ of such a clump of matter can be written as $T(E)=\exp \left(-\tau_{\text {cont }}(E)\right)$. For a worked out example see also Sect. 6.6.

\subsection{Line Absorption}

When light from a background source shines through a clump of matter, a part of the radiation can be absorbed. We discussed already the continuum absorption. The transmission in a spectral line at wavelength $\lambda$ is given by

$$
T(\lambda)=\mathrm{e}^{-\tau(\lambda)}
$$

with

$$
\tau(\lambda)=\tau_{0} \varphi(\lambda)
$$

where $\varphi(\lambda)$ is the line profile and $\tau_{0}$ is the opacity at the line centre $\lambda_{0}$, given by:

$$
\tau_{0}=\frac{\alpha \mathrm{h} \lambda f N_{i}}{2 \sqrt{2 \pi} m_{\mathrm{e}} \sigma_{\mathrm{v}}} .
$$

Apart from the fine structure constant $\alpha$ and Planck's constant h, the optical depth also depends on the properties of the absorber, namely the ionic column density $N_{i}$ and the velocity dispersion $\sigma_{\mathrm{v}}$. Furthermore, it depends on the oscillator strength $f$ which is a dimensionless quantity that is different for each transition and is of order unity for the strongest transitions.

In the simplest approximation, a Gaussian profile $\left.\varphi(\lambda)=\exp -\left(\lambda-\lambda_{0}\right)^{2} / b^{2}\right)$ can be adopted, corresponding to pure Doppler broadening for a thermal plasma. Here $b=\sqrt{2} \sigma$ 
Table 5 As Table 4, but for $\lambda<43 \AA$

\begin{tabular}{|c|c|c|c|c|c|c|c|}
\hline $\begin{array}{l}\mathrm{E} \\
(\mathrm{eV})\end{array}$ & $\begin{array}{l}\lambda \\
(\AA)\end{array}$ & $\begin{array}{l}-\log \\
Q_{\max }\end{array}$ & $\begin{array}{l}\log \\
T_{\max }\end{array}$ & Ion & $\begin{array}{l}\text { Iso-el. } \\
\text { seq. }\end{array}$ & $\begin{array}{l}\text { Lower } \\
\text { level }\end{array}$ & $\begin{array}{l}\text { Upper } \\
\text { level }\end{array}$ \\
\hline 291.52 & 42.530 & 1.32 & 6.18 & $\mathrm{Sx}$ & $\mathrm{N}$ & $2 p^{4} S_{3 / 2}$ & $3 d^{4} P_{5 / 2,3 / 2,1 / 2}$ \\
\hline 298.97 & 41.470 & 1.31 & 5.97 & $\mathrm{Cv}$ & $\mathrm{He}$ & $1 \mathrm{~s}{ }^{1} \mathrm{~S}_{0}$ & $2 s^{3} S_{1}(f)$ \\
\hline 303.07 & 40.910 & 1.75 & 6.29 & Si XII & $\mathrm{Li}$ & $2 s^{2} S_{1 / 2}$ & $3 \mathrm{p}^{2} \mathrm{P}_{3 / 2}$ \\
\hline 307.88 & 40.270 & 1.27 & 5.98 & $\mathrm{Cv}$ & $\mathrm{He}$ & $1 \mathrm{~s}{ }^{1} \mathrm{~S}_{0}$ & $2 \mathrm{p}^{1} \mathrm{P}_{1}(\mathrm{r})$ \\
\hline 315.48 & 39.300 & 1.37 & 6.28 & S XI & $\mathrm{C}$ & $2 p^{3} \mathrm{P}$ & $3 \mathrm{~d}^{3} \mathrm{D},{ }^{1} \mathrm{D},{ }^{3} \mathrm{~F}$ \\
\hline 336.00 & 36.900 & 1.58 & 6.19 & $\mathrm{~S} x$ & $\mathrm{~N}$ & $2 p^{4} S_{3 / 2}$ & $4 d^{4} P_{5 / 2,3 / 2,1 / 2}$ \\
\hline 339.10 & 36.563 & 1.56 & 6.34 & S XII & B & $2 \mathrm{p}^{2} \mathrm{P}_{3 / 2}$ & $3 \mathrm{~d}^{2} \mathrm{D}_{5 / 2}$ \\
\hline 340.63 & 36.398 & 1.56 & 6.34 & S XII & $\mathrm{B}$ & $2 \mathrm{p}^{2} \mathrm{P}_{1 / 2}$ & $3 d^{2} D_{3 / 2}$ \\
\hline 367.47 & 33.740 & 1.47 & 6.13 & C VI & $\mathrm{H}$ & $1 \mathrm{~s}^{2} \mathrm{~S}_{1 / 2}$ & $2 \mathrm{p}^{2} \mathrm{P}_{1 / 2}(\mathrm{Ly} \alpha)$ \\
\hline 367.53 & 33.734 & 1.18 & 6.13 & $\mathrm{CVI}$ & $\mathrm{H}$ & $1 \mathrm{~s}^{2} \mathrm{~S}_{1 / 2}$ & $2 \mathrm{p}^{2} \mathrm{P}_{3 / 2}(\mathrm{Ly} \alpha)$ \\
\hline 430.65 & 28.790 & 1.69 & 6.17 & N VI & $\mathrm{He}$ & $1 \mathrm{~s}{ }^{1} \mathrm{~S}_{0}$ & $2 \mathrm{p}^{1} \mathrm{P}_{1}(\mathrm{r})$ \\
\hline 500.36 & 24.779 & 1.68 & 6.32 & N VII & $\mathrm{H}$ & $1 \mathrm{~s}^{2} \mathrm{~S}_{1 / 2}$ & $2 \mathrm{p}^{2} \mathrm{P}_{3 / 2}(\mathrm{Ly} \alpha)$ \\
\hline 560.98 & 22.101 & 0.86 & 6.32 & O VII & $\mathrm{He}$ & $1 \mathrm{~s}{ }^{1} \mathrm{~S}_{0}$ & $2 s^{3} S_{1}(f)$ \\
\hline 568.55 & 21.807 & 1.45 & 6.32 & O VII & $\mathrm{He}$ & $1 \mathrm{~s}{ }^{1} \mathrm{~S}_{0}$ & $2 \mathrm{p}^{3} \mathrm{P}_{2,1}$ (i) \\
\hline 573.95 & 21.602 & 0.71 & 6.33 & O VII & $\mathrm{He}$ & $1 \mathrm{~s}{ }^{1} \mathrm{~S}_{0}$ & $2 \mathrm{p}^{1} \mathrm{P}_{1}(\mathrm{r})$ \\
\hline 653.49 & 18.973 & 1.05 & 6.49 & O VIII & $\mathrm{H}$ & $1 \mathrm{~s}^{2} \mathrm{~S}_{1 / 2}$ & $2 \mathrm{p}^{2} \mathrm{P}_{1 / 2}(\mathrm{Ly} \alpha)$ \\
\hline 653.68 & 18.967 & 0.77 & 6.48 & O VIII & $\mathrm{H}$ & $1 \mathrm{~s}^{2} \mathrm{~S}_{1 / 2}$ & $2 \mathrm{p}^{2} \mathrm{P}_{3 / 2}(\mathrm{Ly} \alpha)$ \\
\hline 665.62 & 18.627 & 1.58 & 6.34 & O VII & $\mathrm{He}$ & $1 \mathrm{~s}{ }^{1} \mathrm{~S}_{0}$ & $3 p{ }^{1} \mathrm{P}_{1}$ \\
\hline 725.05 & 17.100 & 0.87 & 6.73 & Fe XVII & $\mathrm{Ne}$ & $2 \mathrm{p}^{1} \mathrm{~S}_{0}$ & $3 \mathrm{~s}^{3} \mathrm{P}_{2}$ \\
\hline 726.97 & 17.055 & 0.79 & 6.73 & Fe XVII & $\mathrm{Ne}$ & $2 \mathrm{p}^{1} \mathrm{~S}_{0}$ & $3 s^{3} \mathrm{P}_{1}$ \\
\hline 738.88 & 16.780 & 0.87 & 6.73 & Fe XVII & $\mathrm{Ne}$ & $2 \mathrm{p}^{1} \mathrm{~S}_{0}$ & $3 \mathrm{~s}{ }^{1} \mathrm{P}_{1}$ \\
\hline 771.14 & 16.078 & 1.37 & 6.84 & Fe XVIII & $\mathrm{F}$ & $2 \mathrm{p}^{2} \mathrm{P}_{3 / 2}$ & $3 s^{4} \mathrm{P}_{5 / 2}$ \\
\hline 774.61 & 16.006 & 1.55 & 6.50 & O VIII & $\mathrm{H}$ & $1 \mathrm{~s}^{2} \mathrm{~S}_{1 / 2}$ & $3 \mathrm{p}^{2} \mathrm{P}_{1 / 2,3 / 2}(\operatorname{Ly} \beta)$ \\
\hline 812.21 & 15.265 & 1.12 & 6.74 & Fe XVII & $\mathrm{Ne}$ & $2 \mathrm{p}^{1} \mathrm{~S}_{0}$ & $3 d^{3} D_{1}$ \\
\hline 825.79 & 15.014 & 0.58 & 6.74 & Fe XVII & $\mathrm{Ne}$ & $2 \mathrm{p}^{1} \mathrm{~S}_{0}$ & $3 \mathrm{~d}^{1} \mathrm{P}_{1}$ \\
\hline 862.32 & 14.378 & 1.69 & 6.84 & Fe XVIII & $\mathrm{F}$ & $2 \mathrm{p}^{2} \mathrm{P}_{3 / 2}$ & $3 d^{2} D_{5 / 2}$ \\
\hline 872.39 & 14.212 & 1.54 & 6.84 & Fe XVIII & $\mathrm{F}$ & $2 \mathrm{p}^{2} \mathrm{P}_{3 / 2}$ & $3 d^{2} S_{1 / 2}$ \\
\hline 872.88 & 14.204 & 1.26 & 6.84 & Fe XVIII & $\mathrm{F}$ & $2 \mathrm{p}^{2} \mathrm{P}_{3 / 2}$ & $3 d^{2} D_{5 / 2}$ \\
\hline 896.75 & 13.826 & 1.66 & 6.76 & Fe XVII & $\mathrm{Ne}$ & $2 \mathrm{~s}{ }^{1} \mathrm{~S}_{0}$ & $3 \mathrm{p}^{1} \mathrm{P}_{1}$ \\
\hline 904.99 & 13.700 & 1.61 & 6.59 & $\mathrm{Ne} I X$ & $\mathrm{He}$ & $1 \mathrm{~s}{ }^{1} \mathrm{~S}_{0}$ & $2 s{ }^{3} S_{1}(f)$ \\
\hline 905.08 & 13.699 & 1.61 & 6.59 & Ne IX & $\mathrm{He}$ & $1 \mathrm{~s}{ }^{1} \mathrm{~S}_{0}$ & $2 s{ }^{3} S_{1}(f)$ \\
\hline 916.98 & 13.521 & 1.35 & 6.91 & Fe XIX & $\mathrm{O}$ & $2 \mathrm{p}^{3} \mathrm{P}_{2}$ & $3 d^{3} D_{3}$ \\
\hline 917.93 & 13.507 & 1.68 & 6.91 & Fe XIX & $\mathrm{O}$ & $2 \mathrm{p}^{3} \mathrm{P}_{2}$ & $3 d^{3} P_{2}$ \\
\hline 922.02 & 13.447 & 1.44 & 6.59 & $\mathrm{Ne} I X$ & $\mathrm{He}$ & ${ }^{1 s}{ }^{1} \mathrm{~S}_{0}$ & $2 \mathrm{p}{ }^{1} \mathrm{P}_{1}(\mathrm{r})$ \\
\hline 965.08 & 12.847 & 1.51 & 6.98 & Fe $x x$ & $\mathrm{~N}$ & $2 p^{4} S_{3 / 2}$ & $3 d^{4} P_{5 / 2}$ \\
\hline 966.59 & 12.827 & 1.44 & 6.98 & $\mathrm{Fe} X \mathrm{X}$ & $\mathrm{N}$ & $2 p^{4} S_{3 / 2}$ & $3 d^{4} P_{3 / 2}$ \\
\hline 1009.2 & 12.286 & 1.12 & 7.04 & Fe XXI & $\mathrm{C}$ & $2 \mathrm{p}^{3} \mathrm{P}_{0}$ & $3 \mathrm{~d}^{3} \mathrm{D}_{1}$ \\
\hline 1011.0 & 12.264 & 1.46 & 6.73 & Fe XVII & $\mathrm{Ne}$ & $2 \mathrm{p}^{1} \mathrm{~S}_{0}$ & $4 d^{3} D_{1}$ \\
\hline 1021.5 & 12.137 & 1.77 & 6.77 & $\mathrm{Ne} X$ & $\mathrm{H}$ & $1 \mathrm{~s}^{2} \mathrm{~S}_{1 / 2}$ & $2 \mathrm{p}^{2} \mathrm{P}_{1 / 2}(\mathrm{Ly} \alpha)$ \\
\hline 1022.0 & 12.132 & 1.49 & 6.76 & $\mathrm{Ne} X$ & $\mathrm{H}$ & $1 \mathrm{~s}^{2} \mathrm{~S}_{1 / 2}$ & $2 \mathrm{p}^{2} \mathrm{P}_{3 / 2}(\mathrm{Ly} \alpha)$ \\
\hline 1022.6 & 12.124 & 1.39 & 6.73 & Fe XVII & $\mathrm{Ne}$ & $2 \mathrm{p}^{1} \mathrm{~S}_{0}$ & $4 d{ }^{1} P_{1}$ \\
\hline 1053.4 & 11.770 & 1.38 & 7.10 & Fe XXII & B & $2 p^{2} P_{1 / 2}$ & $3 \mathrm{~d}^{2} \mathrm{D}_{3 / 2}$ \\
\hline
\end{tabular}


Table 5 (Continued)

\begin{tabular}{llllllll}
\hline $\begin{array}{l}\mathrm{E} \\
(\mathrm{eV})\end{array}$ & $\begin{array}{l}\lambda \\
(\AA)\end{array}$ & $\begin{array}{l}-\log \\
Q_{\max }\end{array}$ & $\begin{array}{l}\log \\
T_{\max }\end{array}$ & Ion & $\begin{array}{l}\text { Iso-el. } \\
\text { seq. }\end{array}$ & $\begin{array}{l}\text { Lower } \\
\text { level }\end{array}$ & $\begin{array}{l}\text { Upper } \\
\text { level }\end{array}$ \\
\hline 1056.0 & 11.741 & 1.51 & 7.18 & $\mathrm{Fe}$ XXIII & $\mathrm{Be}$ & $2 \mathrm{p}{ }^{1} \mathrm{P}_{1}$ & $3 \mathrm{~d}{ }^{1} \mathrm{D}_{2}$ \\
1102.0 & 11.251 & 1.73 & 6.74 & $\mathrm{Fe}$ XVII & $\mathrm{Ne}$ & $2 \mathrm{p}{ }^{1} \mathrm{~S}_{0}$ & $5 \mathrm{~d}^{3} \mathrm{D}_{1}$ \\
1352.1 & 9.170 & 1.66 & 6.81 & $\mathrm{Mg} \mathrm{XI}$ & $\mathrm{He}$ & $1 \mathrm{~s}{ }^{1} \mathrm{~S}_{0}$ & $2 \mathrm{p}{ }^{1} \mathrm{P}_{1}(\mathrm{r})$ \\
1472.7 & 8.419 & 1.76 & 7.00 & $\mathrm{Mg} \mathrm{XII}$ & $\mathrm{H}$ & $1 \mathrm{~s}{ }^{2} \mathrm{~S}_{1 / 2}$ & $2 \mathrm{p}^{2} \mathrm{P}_{3 / 2}$ (Ly $\left.\alpha\right)$ \\
1864.9 & 6.648 & 1.59 & 7.01 & $\mathrm{Si} \mathrm{XIII}$ & $\mathrm{He}$ & $1 \mathrm{~s}{ }^{1} \mathrm{~S}_{0}$ & $2 \mathrm{p}^{1} \mathrm{P}_{1}(\mathrm{r})$ \\
2005.9 & 6.181 & 1.72 & 7.21 & $\mathrm{Si} \mathrm{XIV}$ & $\mathrm{H}$ & $1 \mathrm{~s}{ }^{2} \mathrm{~S}_{1 / 2}$ & $2 \mathrm{p}^{2} \mathrm{P}_{3 / 2}$ (Ly $\left.\alpha\right)$ \\
6698.6 & 1.851 & 1.43 & 7.84 & Fe XXV & $\mathrm{He}$ & $1 \mathrm{~s}{ }^{1} \mathrm{~S}_{0}$ & $2 \mathrm{p}{ }^{1} \mathrm{P}_{1}(\mathrm{r})$ \\
6973.1 & 1.778 & 1.66 & 8.17 & Fe XXVI & $\mathrm{H}$ & $1 \mathrm{~s}{ }^{2} \mathrm{~S}_{1 / 2}$ & $2 \mathrm{p}{ }^{2} \mathrm{P}_{3 / 2}$ (Ly $\left.\alpha\right)$ \\
\hline
\end{tabular}

with $\sigma$ the normal Gaussian root-mean-square width. The full width at half maximum of this profile is given by $\sqrt{\ln 256} \sigma$ or approximately $2.35 \sigma$. One may even include a turbulent velocity $\sigma_{\mathrm{t}}$ into the velocity width $\sigma_{\mathrm{v}}$, such that

$$
\sigma_{\mathrm{v}}^{2}=\sigma_{\mathrm{t}}^{2}+k T / m_{i}
$$

with $m_{i}$ the mass of the ion (we have tacitly changed here from wavelength to velocity units through the scaling $\left.\Delta \lambda / \lambda_{0}=\Delta v / \mathrm{c}\right)$.

The equivalent width $W$ of the line is calculated from

$$
W=\frac{\lambda \sigma}{\mathrm{c}} \int_{-\infty}^{\infty}\left[1-\exp \left(-\tau_{0} e^{-y^{2} / 2}\right)\right] \mathrm{d} y .
$$

For the simple case that $\tau_{0} \ll 1$, the integral can be evaluated as

$$
\tau_{0} \ll 1: \quad W=\frac{\alpha \mathrm{h} \lambda^{2} f N_{i}}{2 m_{\mathrm{e}} \mathrm{c}}=\frac{1}{2} \alpha \frac{\mathrm{h} v}{m_{\mathrm{e}} \mathrm{c}^{2}} f \lambda^{2} N_{i} .
$$

A Gaussian line profile is only a good approximation when the Doppler width of the line is larger than the natural width of the line. The natural line profile for an absorption line is a Lorentz profile $\varphi(\lambda)=1 /\left(1+x^{2}\right)$ with $x=4 \pi \Delta v / A$. Here $\Delta v$ is the frequency difference $v-v_{0}$ and $A$ is the total transition probability from the upper level downwards to any level, including all radiative and Auger decay channels.

Convolving the intrinsic Lorentz profile with the Gaussian Doppler profile due to the thermal or turbulent motion of the plasma, gives the well-known Voigt line profile

$$
\varphi=H(a, y)
$$

where

$$
a=A \lambda / 4 \pi b
$$

and $y=\mathrm{c} \Delta \lambda / b \lambda$. The dimensionless parameter $a$ (not to be confused with the $a$ in (4)) represents the relative importance of the Lorentzian term $(\sim A)$ to the Gaussian term $(\sim b)$. It should be noted here that formally for $a>0$ the parameter $\tau_{0}$ defined by (44) is not exactly the optical depth at line centre, but as long as $a \ll 1$ it is a fair approximation. For $a \gg 1$ it can be shown that $H(a, 0) \rightarrow 1 / a \sqrt{\pi}$. 


\subsection{Some Important X-ray Absorption Lines}

There are several types of absorption lines. The most well-known are the normal strong resonance lines, which involve electrons from the outermost atomic shells. Examples are the $1 \mathrm{~s}-2 \mathrm{p}$ line of O VII at $21.60 \AA$, the O VIII Ly $\alpha$ doublet at $18.97 \AA$ and the well known 2s-2p doublet of O VI at 1032 and $1038 \AA$. See Richter et al. 2008-Chap. 3, this issue, for an extensive discussion on these absorption lines in the WHIM.

The other class are the inner-shell absorption lines. In this case the excited atom is often in an unstable state, with a large probability for the Auger process (Sect. 3.2.4). As a result, the parameter $a$ entering the Voigt profile (49) is large and therefore the lines can have strong damping wings.

In some cases the lines are isolated and well resolved, like the O VII and O VIII $1 \mathrm{~s}-2 \mathrm{p}$ lines mentioned above. However, in other cases the lines may be strongly blended, like for the higher principal quantum number transitions of any ion in case of high column densities. Another important group of strongly blended lines are the inner-shell transitions of heavier elements like iron. They form so-called unresolved transition arrays (UTAs); the individual lines are no longer recognisable. The first detection of these UTAs in astrophysical sources was in a quasar (Sako et al. 2001).

In Table 6 we list the 70 most important X-ray absorption lines for $\lambda<100 \AA$. The importance was determined by calculating the maximum ratio $W / \lambda$ that these lines reach for any given temperature.

Note the dominance of lines from all ionisation stages of oxygen in the 17-24 $\AA$ band. Furthermore, the Fe IX and Fe XVII lines are the strongest iron features. These lines are weaker than the oxygen lines because of the lower abundance of iron; they are stronger than their neighbouring iron ions because of somewhat higher oscillator strengths and a relatively higher ion fraction (cf. Fig. 7).

Note that the strength of these lines can depend on several other parameters, like turbulent broadening, deviations from CIE, saturation for higher column densities, etc., so some care should be taken when the strongest line for other physical conditions are sought.

\subsection{Curve of Growth}

In most practical situations, X-ray absorption lines are unresolved or poorly resolved. As a consequence, the physical information about the source can only be retrieved from basic parameters such as the line centroid (for Doppler shifts) and equivalent width $W$ (as a measure of the line strength).

As outlined in Sect. 6.3, the equivalent width $W$ of a spectral line from an ion $i$ depends, apart from atomic parameters, only on the column density $N_{i}$ and the velocity broadening $\sigma_{\mathrm{v}}$. A useful diagram is the so-called curve of growth. Here we present it in the form of a curve giving $W$ versus $N_{i}$ for a fixed value of $\sigma_{\mathrm{v}}$.

The curve of growth has three regimes. For low column densities, the optical depth at line centre $\tau_{0}$ is small, and $W$ increases linearly with $N_{i}$, independent of $\sigma_{\mathrm{v}}(47)$. For increasing column density, $\tau_{0}$ starts to exceed unity and then $W$ increases only logarithmically with $N_{i}$. Where this happens exactly, depends on $\sigma_{\mathrm{v}}$. In this intermediate logarithmic branch $W$ depends both on $\sigma_{\mathrm{v}}$ and $N_{i}$, and hence by comparing the measured $W$ with other lines from the same ion, both parameters can be estimated. At even higher column densities, the effective opacity increases faster (proportional to $\sqrt{N_{i}}$ ), because of the Lorentzian line wings. Where this happens depends on the Voigt parameter $a$ (49). In this range of densities, $W$ does not depend any more on $\sigma_{\mathrm{v}}$. 
Table 6 The most important X-ray absorption lines with $\lambda<100 \AA$. Calculations are done for a plasma in CIE with proto-solar abundances (Lodders 2003) and only thermal broadening. The calculations are done for a hydrogen column density of $10^{24} \mathrm{~m}^{-2}$, and we list equivalent widths for the temperature $T_{\max }$ (in $\mathrm{K}$ ) where it reaches a maximum. Equivalent widths are calculated for the listed line only, not taking into account blending by other lines. For saturated, overlapping lines, like the O VIII Ly $\alpha$ doublet near $18.97 \AA$, the combined equivalent width can be smaller than the sum of the individual equivalent widths

\begin{tabular}{|c|c|c|c|c|c|c|c|}
\hline $\begin{array}{l}\lambda \\
(\AA)\end{array}$ & Ion & $\begin{array}{l}W_{\max } \\
(\mathrm{m} \AA)\end{array}$ & $\begin{array}{l}\log \\
T_{\max }\end{array}$ & $\begin{array}{l}\lambda \\
(\AA)\end{array}$ & Ion & $\begin{array}{l}W_{\max } \\
(\mathrm{m} \AA)\end{array}$ & $\begin{array}{l}\log \\
T_{\max }\end{array}$ \\
\hline 9.169 & Mg XI & 1.7 & 6.52 & 31.287 & $\mathrm{~N}_{\mathrm{I}}$ & 5.5 & 4.04 \\
\hline 13.447 & $\mathrm{Ne} I X$ & 4.6 & 6.38 & 33.426 & $\mathrm{CV}$ & 7.0 & 5.76 \\
\hline 13.814 & $\mathrm{Ne}$ VII & 2.2 & 5.72 & 33.734 & C VI & 12.9 & 6.05 \\
\hline 15.014 & Fe XVII & 5.4 & 6.67 & 33.740 & C VI & 10.3 & 6.03 \\
\hline 15.265 & Fe XVII & 2.2 & 6.63 & 34.973 & $\mathrm{CV}$ & 10.3 & 5.82 \\
\hline 15.316 & $\mathrm{FeXV}$ & 2.3 & 6.32 & 39.240 & S XI & 6.5 & 6.25 \\
\hline 16.006 & O VIII & 2.6 & 6.37 & 40.268 & $\mathrm{C} \mathrm{V}$ & 17.0 & 5.89 \\
\hline 16.510 & $\mathrm{Fe} I X$ & 5.6 & 5.81 & 40.940 & C IV & 7.2 & 5.02 \\
\hline 16.773 & $\mathrm{Fe} I \mathrm{X}$ & 3.0 & 5.81 & 41.420 & C IV & 14.9 & 5.03 \\
\hline 17.396 & O VII & 2.8 & 6.06 & 42.543 & S X & 6.3 & 6.14 \\
\hline 17.768 & O VII & 4.4 & 6.11 & 50.524 & SIX & 11.0 & 6.14 \\
\hline 18.629 & O VII & 6.9 & 6.19 & 51.807 & S VII & 7.6 & 5.68 \\
\hline 18.967 & O VIII & 8.7 & 6.41 & 55.305 & SI IX & 14.0 & 6.06 \\
\hline 18.973 & O VIII & 6.5 & 6.39 & 60.161 & S VII & 12.2 & 5.71 \\
\hline 19.924 & $\mathrm{OV}$ & 3.2 & 5.41 & 61.019 & SI VIII & 11.9 & 5.92 \\
\hline 21.602 & O VII & 12.1 & 6.27 & 61.070 & SI VIII & 13.2 & 5.93 \\
\hline 22.006 & O VI & 5.1 & 5.48 & 62.751 & Mg IX & 11.1 & 5.99 \\
\hline 22.008 & O VI & 4.1 & 5.48 & 68.148 & SI VII & 10.1 & 5.78 \\
\hline 22.370 & $\mathrm{OV}$ & 5.4 & 5.41 & 69.664 & SI VII & 10.9 & 5.78 \\
\hline 22.571 & O IV & 3.6 & 5.22 & 70.027 & SI VII & 11.3 & 5.78 \\
\hline 22.739 & O IV & 5.1 & 5.24 & 73.123 & SI VII & 10.7 & 5.78 \\
\hline 22.741 & O IV & 7.2 & 5.23 & 74.858 & Mg VIII & 17.1 & 5.93 \\
\hline 22.777 & O IV & 13.8 & 5.22 & 80.449 & SI VI & 13.0 & 5.62 \\
\hline 22.978 & O III & 9.4 & 4.95 & 80.577 & SI VI & 12.7 & 5.61 \\
\hline 23.049 & O III & 7.1 & 4.96 & 82.430 & Fe IX & 14.0 & 5.91 \\
\hline 23.109 & O III & 15.1 & 4.95 & 83.128 & SI VI & 14.2 & 5.63 \\
\hline 23.350 & O II & 7.5 & 4.48 & 83.511 & Mg VII & 14.2 & 5.82 \\
\hline 23.351 & O II & 12.6 & 4.48 & 83.910 & Mg VII & 19.8 & 5.85 \\
\hline 23.352 & O II & 16.5 & 4.48 & 88.079 & $\mathrm{Ne}$ VIII & 15.2 & 5.80 \\
\hline 23.510 & O I & 7.2 & 4.00 & 95.385 & Mg VI & 15.5 & 5.67 \\
\hline 23.511 & O I & 15.0 & 4.00 & 95.421 & Mg VI & 18.7 & 5.69 \\
\hline 24.779 & N VII & 4.6 & 6.20 & 95.483 & Mg VI & 20.5 & 5.70 \\
\hline 24.900 & N VI & 4.0 & 5.90 & 96.440 & SI V & 16.8 & 5.33 \\
\hline 28.465 & C VI & 4.6 & 6.01 & 97.495 & Ne VII & 22.8 & 5.74 \\
\hline 28.787 & N VI & 9.8 & 6.01 & 98.131 & $\mathrm{Ne}$ VI & 16.7 & 5.65 \\
\hline
\end{tabular}

In Fig. 10 we show a few characteristic examples. The examples of O I and O vi illustrate the higher $W$ for UV lines as compared to X-ray lines (cf. (47)) as well as the fact that inner shell transitions (in this case the X-ray lines) have larger $a$-values and hence reach sooner the 

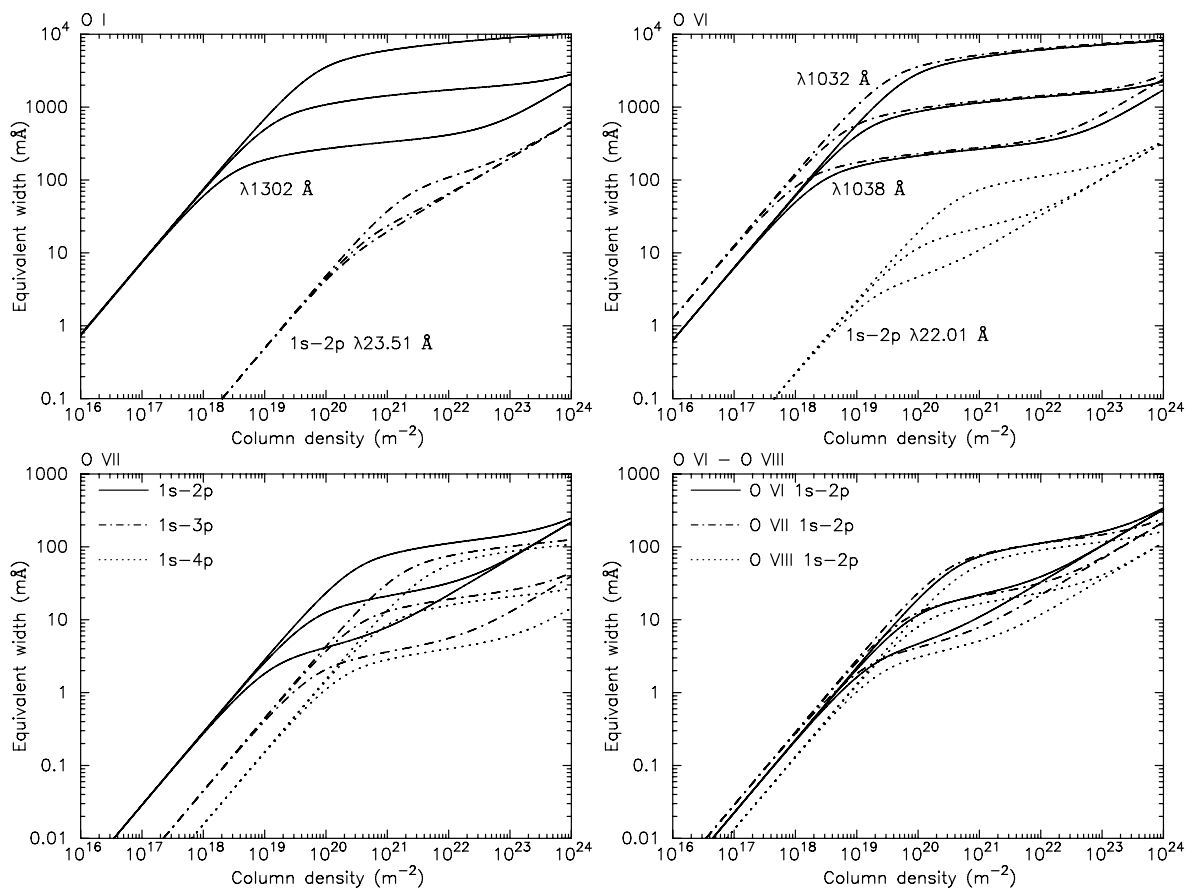

Fig. 10 Equivalent width versus column density for a few selected oxygen absorption lines. The curves have been calculated for Gaussian velocity dispersions $\sigma=b / \sqrt{2}$ of 10,50 and $250 \mathrm{~km} \mathrm{~s}^{-1}$, from bottom to top for each line. Different spectral lines are indicated with different line styles

square root branch. The example of O VII shows how different lines from the same ion have different equivalent width ratios depending on $\sigma_{\mathrm{v}}$, hence offer an opportunity to determine that parameter. The last frame shows the (non)similarities for similar transitions in different ions. The innershell nature of the transition in O vI yields a higher $a$ value and hence an earlier onset of the square root branch.

\subsection{Galactic Foreground Absorption}

All radiation from X-ray sources external to our own Galaxy has to pass through the interstellar medium of our Galaxy, and the intensity is reduced by a factor of $\mathrm{e}^{-\tau(E)}$ with the optical depth $\tau(E)=\sum_{i} \sigma_{i}(E) \int n_{i}(\ell) \mathrm{d} \ell$, with the summation over all relevant ions $i$ and the integration over the line of sight $\mathrm{d} \ell$. The absorption cross section $\sigma_{i}(E)$ is often taken to be simply the (continuum) photoionisation cross section, but for high-resolution spectra it is necessary to include also the line opacity, and for very large column densities also other processes such as Compton ionisation or Thomson scattering.

For a cool, neutral plasma with cosmic abundances one often writes

$$
\tau=\sigma_{\mathrm{eff}}(E) N_{\mathrm{H}}
$$

where the hydrogen column density $N_{\mathrm{H}} \equiv \int n_{\mathrm{H}} \mathrm{d} x$. In $\sigma_{\text {eff }}(E)$ all contributions to the absorption from all elements as well as their abundances are taken into account. 

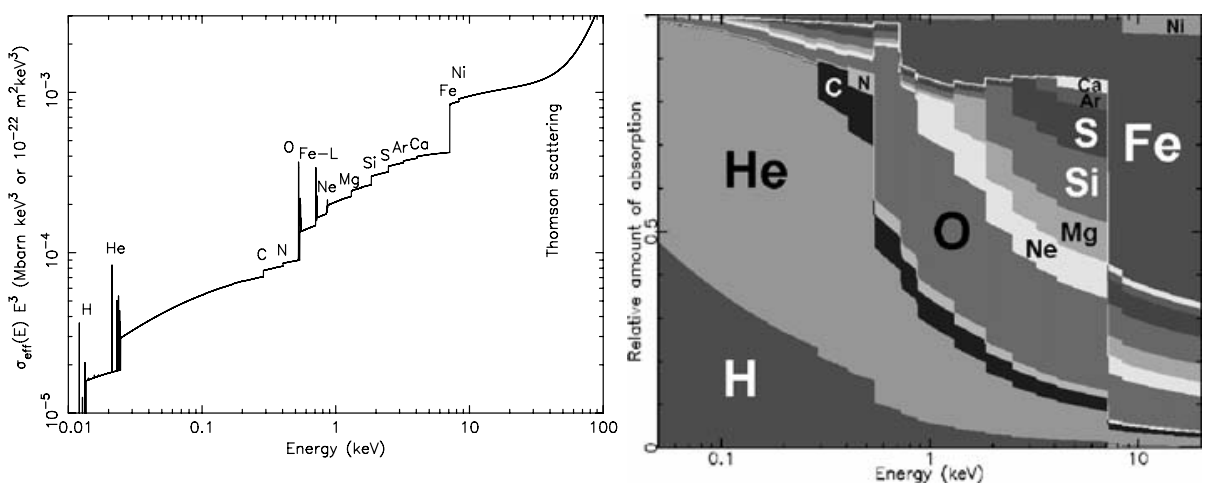

Fig. 11 Left panel: Neutral interstellar absorption cross section per hydrogen atom, scaled with $E^{3}$. The most important edges with associated absorption lines are indicated, as well as the onset of Thompson scattering around $30 \mathrm{keV}$. Right panel: Contribution of the various elements to the total absorption cross section as a function of energy, for Solar abundances
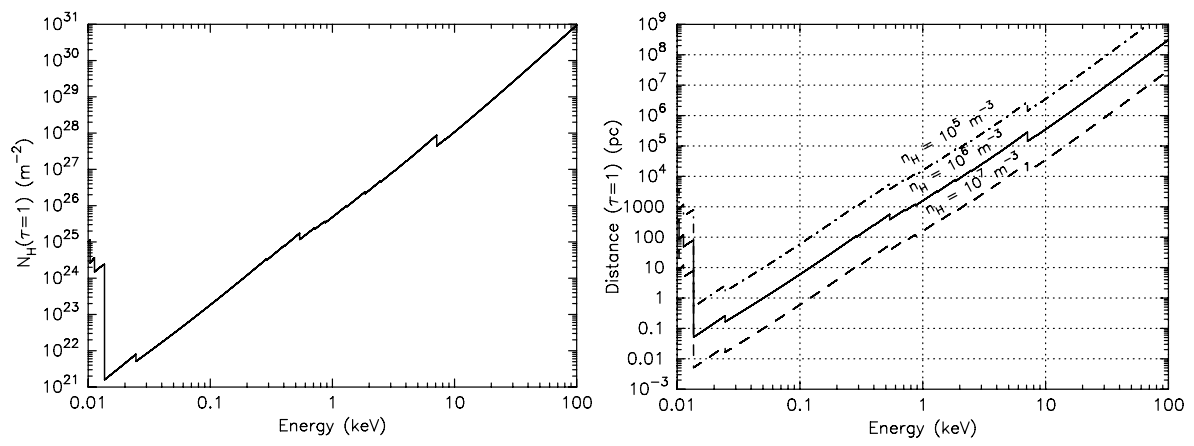

Fig. 12 Left panel: Column density for which the optical depth becomes unity. Right panel: Distance for which $\tau=1$ for three characteristic densities

The relative contribution of the elements is also made clear in Fig. 11. Below $0.28 \mathrm{keV}$ (the carbon edge) hydrogen and helium dominate, while above $0.5 \mathrm{keV}$ in particular oxygen is important. At the highest energies, above $7.1 \mathrm{keV}$, iron is the main opacity source.

Yet another way to represent these data is to plot the column density or distance for which the optical depth becomes unity (Fig. 12). This figure shows that in particular at the lowest energies X-rays are most strongly absorbed. The visibility in that region is thus very limited. Below $0.2 \mathrm{keV}$ it is extremely hard to look outside our Milky Way.

The interstellar medium is by no means a homogeneous, neutral, atomic gas. In fact, it is a collection of regions all with different physical state and composition. This affects its $\mathrm{X}$-ray opacity. We briefly discuss here some of the most important features.

The ISM contains cold gas $(<50 \mathrm{~K})$, warm, neutral or lowly ionised gas $(6000-10000 \mathrm{~K})$ as well as hotter gas (a few million K). We have seen before (see Sect. 3.2.2) that for ions the absorption edges shift to higher energies for higher ionisation. Thus, with instruments of sufficient spectral resolution, the degree of ionisation of the ISM can be deduced from the relative intensities of the absorption edges. Cases with such high column densities are rare, however (and only occur in some AGN outflows), and for the bulk of the ISM the column density of the ionised ISM is low enough that only the narrow absorption lines are visible 

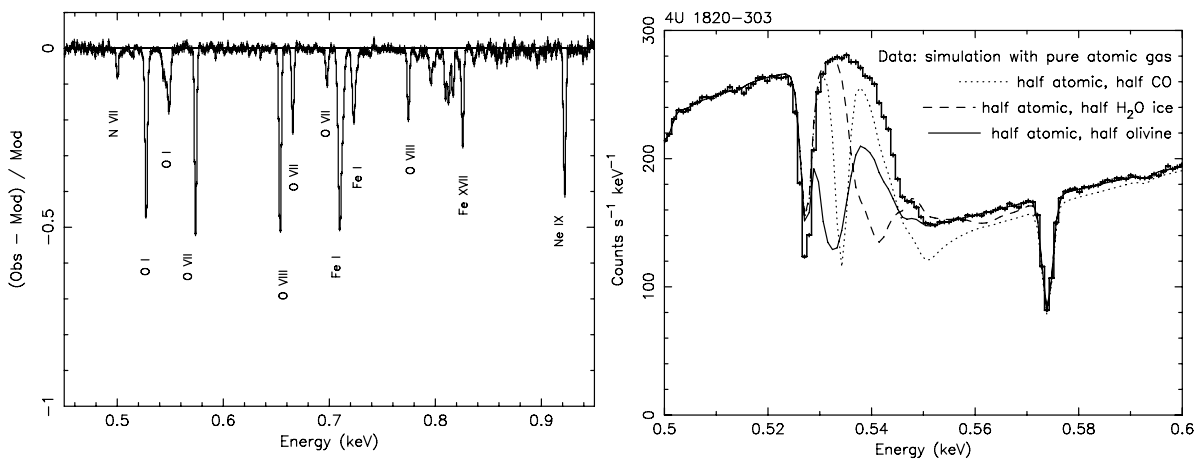

Fig. 13 Left panel: Simulated $100 \mathrm{ks}$ absorption spectrum as observed with the Explorer of Diffuse Emission and Gamma-ray Burst Explosions (EDGE), a mission proposed for ESA's Cosmic Vision program. The parameters of the simulated source are similar to those of 4U 1820-303 (Yao and Wang 2006). The plot shows the residuals of the simulated spectrum if the absorption lines in the model are ignored. Several characteristic absorption features of both neutral and ionised gas are indicated.

Right panel: Simulated spectrum for the X-ray binary 4U 1820-303 for $100 \mathrm{ks}$ with the WFS instrument of EDGE. The simulation was done for all absorbing oxygen in its pure atomic state, the models plotted with different line styles show cases where half of the oxygen is bound in $\mathrm{CO}$, water ice or olivine. Note the effective shift of the absorption edge and the different fine structure near the edge. All of this is well resolved by EDGE, allowing a determination of the molecular composition of dust in the line of sight towards this source. The absorption line at 0.574 is due to highly ionised O VII

(see Fig. 9). These lines are only visible when high spectral resolution is used (see Fig. 13). It is important to recognise these lines, as they should not be confused with absorption line from within the source itself. Fortunately, with high spectral resolution the cosmologically redshifted absorption lines from the X-ray source are separated well from the foreground hot ISM absorption lines. Only for lines from the Local Group this is not possible, and the situation is here complicated as the expected temperature range for the diffuse gas within the Local Group is similar to the temperature of the hot ISM.

Another ISM component is dust. A significant fraction of some atoms can be bound in dust grains with varying sizes, as shown below (from Wilms et al. 2000):

$\begin{array}{ccccccccccccc}\mathrm{H} & \mathrm{He} & \mathrm{C} & \mathrm{N} & \mathrm{O} & \mathrm{Ne} & \mathrm{Mg} & \mathrm{Si} & \mathrm{S} & \mathrm{Ar} & \mathrm{Ca} & \mathrm{Fe} & \mathrm{Ni} \\ 0 & 0 & 0.5 & 0 & 0.4 & 0 & 0.8 & 0.9 & 0.4 & 0 & 0.997 & 0.7 & 0.96\end{array}$

The numbers represent the fraction of the atoms that are bound in dust grains. Noble gases like $\mathrm{Ne}$ and $\mathrm{Ar}$ are chemically inert hence are generally not bound in dust grains, but other elements like $\mathrm{Ca}$ exist predominantly in dust grains. Dust has significantly different X-ray properties compared to gas or hot plasma. First, due to the chemical binding, energy levels are broadened significantly or even absent. For example, for oxygen in most bound forms (like $\mathrm{H}_{2} \mathrm{O}$ ) the remaining two vacancies in the $2 \mathrm{p}$ shell are effectively filled by the two electrons from the other bound atom(s) in the molecule. Therefore, the strong $1 \mathrm{~s}-2 \mathrm{p}$ absorption line at $23.51 \AA(527 \mathrm{eV})$ is not allowed when the oxygen is bound in dust or molecules, because there is no vacancy in the $2 p$ shell. Transitions to higher shells such as the $3 p$ shell are possible, however, but these are blurred significantly and often shifted due to the interactions in the molecule. Each constituent has its own fine structure near the K-edge (Fig. 13, right panel). This fine structure offers therefore the opportunity to study the (true) chemical composition of the dust, but it should be said that the details of the edges in different important compounds are not always (accurately) known, and can differ depending on the state: for 
Fig. 14 Typical Cosmic X-ray background spectrum. The thick solid line indicates the total spectrum, the dashed line the absorbed power law contribution due to unresolved point sources. The contribution of the local hot bubble is indicated by the thin, grey line. It dominates the line emission below $\sim 0.2 \mathrm{keV}$. The other thermal components are not indicated separately, but they are included in the total; they contribute most of the line flux above $0.2 \mathrm{keV}$. The calculation is done with a spectral resolution of $1 \mathrm{eV}$

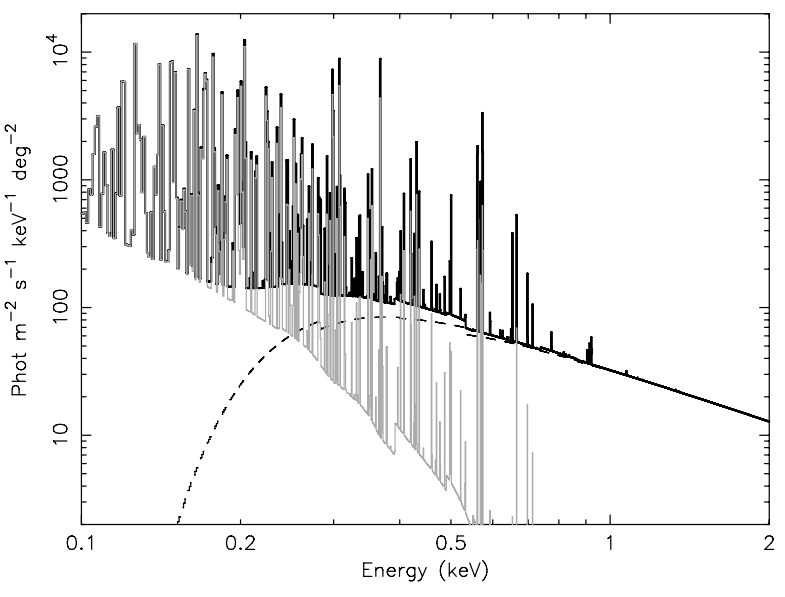

example water, crystalline and amorphous ice all have different characteristics. On the other hand, the large scale edge structure, in particular when observed at low spectral resolution, is not so much affected. For sufficiently high column densities of dust, self-shielding within the grains should be taken into account, and this reduces the average opacity per atom.

Finally, we mention here that dust also causes scattering of X-rays. This is in particular important for higher column densities. For example, for the Crab nebula $\left(N_{\mathrm{H}}=\right.$ $3.2 \times 10^{25} \mathrm{~m}^{-2}$ ), at an energy of $1 \mathrm{keV}$ about $10 \%$ of all photons are scattered in a halo, of which the widest tails have been observed out to a radius of at least half a degree; the scattered fraction increases with increasing wavelength.

\section{Galactic Foreground Emission}

The interstellar medium of our Galaxy is a complex medium. While all phases of the ISM can be seen in X-ray absorption towards bright X-ray sources (see previous section), only the hot phase is seen in emission and contributes to the cosmic X-ray background.

This cosmic X-ray background has different components. Kuntz and Snowden (2000) distinguish four different components as outlined below. First, there is an absorbed powerlaw like component, consisting mostly of unresolved extragalactic point sources. With high spatial resolution like for example available on Chandra, a large part of this component can be resolved into the individual point sources. The second component is the so-called Local Hot Bubble, most likely a large, old supernova remnant embedding our Solar system. The million degree gas of this diffuse medium is almost unabsorbed, as it is nearby and there is little intervening neutral gas. Finally, there are the soft and hard diffuse components, consisting of absorbed, diffuse thermal emission which arises from the disk of our Galaxy but may also contain contributions from a Galactic halo and distant WHIM emission.

Figure 14 shows a simulated spectrum of the total X-ray background. Note however that the details of this spectrum may differ significantly for different parts of the sky. There are variations on large scales, from the Galactic plane to the poles. There are also smaller scale variations. For instance, there are large loops corresponding to old supernova remnants or (super)bubbles, and large molecular cloud complexes obscuring the more distant background components. 
Fig. 15 Cooling curves $\Lambda(T)$ for plasmas of different composition, under CIE conditions. From top to bottom: $1,0.3,0.1,0.01$ and 0.001 times Solar abundances. After Sutherland and Dopita (1993)

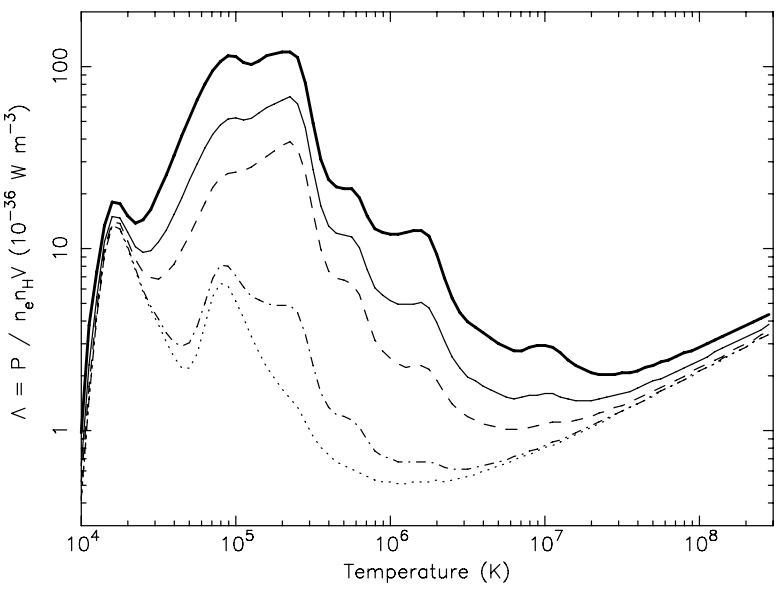

\section{Cooling Function}

The cooling of a plasma by thermal radiation is usually expressed by the cooling function $\Lambda(T)$. This is essentially the bolometric power $P$ emitted as thermal radiation by the plasma, normalised by the emission measure $Y=n_{\mathrm{e}} n_{\mathrm{H}} V$. Accordingly, the characteristic cooling time, defined as the thermal energy of the plasma divided by the emitted power, $t_{\text {cool }}=$ $\frac{3}{2}\left(1+n_{\mathrm{i}} / n_{\mathrm{e}}\right) \mathrm{k} T / \Lambda n_{\mathrm{H}}$.

Cooling is important for the evolution of several plasmas such as the cool cores of clusters but also for the global evolution of the WHIM. A set of useful cooling curves are given by Sutherland and Dopita (1993). An example is shown in Fig. 15. These are valid under CIE conditions. When photoionisation is important, modifications are needed. Here we have normalised $\Lambda$ to the emission measure of the plasma, but note that sometimes other scalings are being used (for example expressions containing the ion density $n_{\mathrm{i}}$ or $n_{\mathrm{e}}^{2}$ ). Note that the Solar abundances used in Fig. 15 are the older values of Anders and Grevesse (1989) which differ significantly from more recent estimates, for example Lodders (2003), in particular for important coolants such as iron (new values 26\% lower) and oxygen (new values $32 \%$ lower). The big hump at low temperatures is produced mainly by line radiation, while the high temperature $\sim T^{0.5}$ tail above $10^{7} \mathrm{~K}$ is mainly produced by Bremsstrahlung.

Finally, a more recent set of cooling curves, suited in particular for lower temperatures and primordial gas, was published by Maio et al. (2007).

\section{Ionisation and Excitation by Non-thermal Electrons}

In most cases, astrophysical plasmas are modelled using Maxwellian velocity distributions, even under NEI or PIE conditions. Often this is justified, but there are situations where deviations from a Maxwellian distribution occur. Examples are accelerated electrons penetrating cooler regions, as may occur in or near shocks or flares. We refrain here from a full discussion, but only mention here that spectral codes such as SPEX (Sect. 10.1) allow the calculation of the ionisation balance and the emanating spectrum under such circumstances, provided the true electron distribution can be approximated by the sum of non-relativistic Maxwellians. When there are strong relativistic electron contributions, this approach cannot be used anymore as here relativistic corrections to the cross sections are needed, which are usually not taken into account in most plasma codes. 


\section{Plasma Modelling}

\subsection{Available Codes}

There are several spectral codes available that can be used to model hot plasmas. Without being complete, we mention here briefly the following codes. For a more extensive review, see Raymond (2005).

First, there are codes which calculate predominantly spectra in Collisional Ionisation Equilibrium:

1. CHIANTI ${ }^{1}$ (named after the wine region near Firenze, Landi et al. 2006) evolved from early work by Landini and Monsignori Fossi (1970); originally, it focused on the calculation of Solar spectra and hence EUV lines are well represented in this code.

2. APEC $^{2}$ (Astrophysical Plasma Emission Code (Smith et al. 2001) evolved from the original Raymond and Smith (1977) code; originally, the main goal was to calculate the emissivity and cooling curve properly. This model is available in the popular fitting package XSPEC $^{3}$ (Arnaud 1996).

3. Mekal (after MEwe, KAastra \& Liedahl; see Mewe et al. 1995) evolved from early work by Mewe and collaborators starting from 1970 onwards. The Mekal model is also incorporated in XSPEC.

The Mekal model is now part of the SPEX package, ${ }^{4}$ (Kaastra et al. 1996b). SPEX is a spectral fitting package developed at SRON Utrecht based on the original CIE models of Mewe and collaborators. It has grown significantly and also contains NEI and a few simple PIE models; more models are under development. The package offers the opportunity to plot or tabulate also various physical parameters of interest of the model, apart from the spectrum, and the atomic physics used in the Mekal model has been significantly updated in the SPEX version as compared to the older version available in XSPEC. A majority of all plots shown in this paper were produced using SPEX.

There are also models focusing upon photo-ionised plasmas. We mention here two codes (apart from SPEX):

1. XSTAR ${ }^{5}$ evolved from the original work of Kallman and McCray (1982), focusing upon the X-ray band. It is relatively straightforward to use and there are options to use it with XSPEC.

2. Cloudy ${ }^{6}$ (Ferland et al. 1998) evolved from work by Ferland and collaborators that originally was meant to model optical emission line regions. It gradually evolved to the X-ray band. It is somewhat more complicated to use, but has more options than XSTAR.

\subsection{Important Parameters}

In order to calculate X-ray spectra for collisional plasmas, there is a set of parameters that determine the spectral shape and flux. The two most important parameters are the electron

\footnotetext{
${ }^{1}$ http://www.arcetri.astro.it/science/chianti/chianti.html.

${ }^{2}$ http://cxc.harvard.edu/atomdb.

${ }^{3}$ http://heasarc.gsfc.nasa.gov/docs/xanadu/xspec.

${ }^{4}$ http://www.sron.nl/spex.

${ }^{5}$ http://heasarc.gsfc.nasa.gov/docs/software/xstar/xstar.html.

${ }^{6}$ http://www.nublado.org.
} 
temperature $T_{\mathrm{e}}$ and emission measure $Y$ of the gas. The emission measure is sometimes not well or even incorrectly defined. We follow here the definition used in SPEX, namely $Y=\int n_{\mathrm{e}} n_{\mathrm{H}} \mathrm{d} V$ where $n_{\mathrm{e}}$ and $n_{\mathrm{H}}$ are the electron and hydrogen density (whether ionised or not). Sometimes people use " $n^{2}$ " in this expression, but it is not always obvious whether ion density, baryon density, electron density, total particle density or something else is meant. The next important parameters are the abundances of the elements; these do not only determine the line emission spectrum, but also affect the continuum emission (Sect. 5.1). Note that the abundances also affect the precise values of $n_{\mathrm{e}} / n_{\mathrm{H}}$ and $n_{\mathrm{i}} / n_{\mathrm{H}}$. For a fully ionised plasma with proto-solar abundances (Lodders 2003) these ratios are 1.201 and 1.097, respectively (for the older Anders and Grevesse (1989) abundances these ratios are 1.209 and 1.099).

For ionising plasmas (NEI) also the parameter $U=\int n_{\mathrm{e}} \mathrm{d} t$ (see (28)) is important, as this describes the evolution of a shocked or recombining gas towards equilibrium.

When high spectral resolution observations are available, other parameters are important, like the ion temperature and turbulent velocity. These parameters determine the width of the spectral lines (see Sect. 5.2.2 and Sect. 6.3). Note that in non-equilibrium plasmas, the electron and ion temperatures are not necessarily the same. In an extreme case, the supernova remnant SN 1006, Vink et al. (2003) measured an oxygen ion temperature that is 350 times larger than the electron temperature of $1.5 \mathrm{keV}$. SPEX has also options to approximate the spectra when there are non-Maxwellian tails to the electron distribution (Sect. 9). These tails may be produced by non-thermal acceleration mechanisms (Petrosian et al. 2008-Chap. 9, this issue).

In general, for the low densities encountered in the diffuse ISM of galaxies, in clusters and in the WHIM, the absolute electron density is not important (except for the overall spectral normalisation through the emission measure $Y$ ). Only in the higher density regions of for example stars $\mathrm{X}$-ray line ratios are affected by density effects, but this is outside the scope of the present paper.

However, if the density becomes extremely low, such as in the lowest density parts of the WHIM, the gas density matters, as in such situations photoionisation by the diffuse radiation field of galaxies or the cosmic background becomes important. But even in such cases, it is not the spectral shape but the ionisation (im)balance that is affected most. In those cases essentially the ratio of incoming radiation flux to gas density determines the photoionisation equilibrium.

\subsection{Multi-Temperature Emission and Absorption}

Several astrophysical sources cannot be characterised by a single temperature. There may be various reasons for this. It may be that the source has regions with different temperatures that cannot be spatially resolved due to the large distance to the source, the low angular resolution of the X-ray instrument, or superposition of spectra along the line of sight. Examples of these three conditions are stellar coronae (almost point sources as seen from Earth), integrated cluster of galaxies spectra, or spectra along the line of sight through the cooling core of a cluster, respectively. But also in other less obvious cases multi-temperature structure can be present. For example Kaastra et al. (2004) showed using deprojected spectra that at each position in several cool core clusters there is an intrinsic temperature distribution. This may be due to unresolved smaller regions in pressure equilibrium with different densities and hence different temperatures.

The most simple solution is to add as many temperature components as are needed to describe the observed spectrum, but there are potential dangers to this. The main reason is 

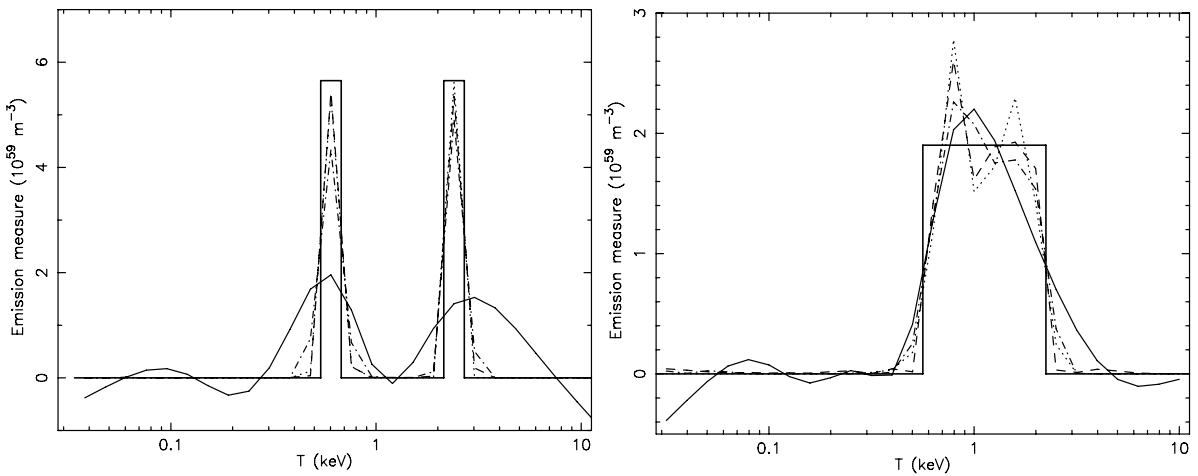

Fig. 16 Reconstructed DEM distribution for a simulated spectrum containing two equally strong isothermal components of 0.6 and $2.4 \mathrm{keV}$ (left panel) or a uniform distribution between 0.6 and $2.4 \mathrm{keV}$ (right panel). Thick solid line: the input model; thin solid line: regularisation method; dot-dashed line: polynomial method; dashed line: genetic algorithm; dotted line: two broadened Gaussian components. The corresponding best-fit spectra differ in $\chi^{2}$ by less than 2 for each case. Obviously, small scale detail cannot be recovered but bulk properties such as the difference between a bimodal DEM distribution (left panel) or a flat distribution (right panel) can be recovered. From (Kaastra et al. 1996a). See that paper for more details

temperature sensitivity. Most spectral temperature indicators (line fluxes, Bremsstrahlung exponential cut-off) change significantly when the temperature is changed by a factor of two. However, for smaller temperature changes, the corresponding spectral changes are also minor. For example, a source with two components of equal emission measure $Y$ and $\mathrm{k} T=0.99$ and $1.01 \mathrm{keV}$, respectively, is almost indistinguishable from a source with the emission measure $2 Y$ and temperature $1.00 \mathrm{keV}$. Obviously, the most general case is a continuous differential emission measure distribution (DEM) $D(T) \equiv \mathrm{d} Y(T) / \mathrm{d} T$. Typically, in each logarithmic temperature range with a width of a factor of two, one can redistribute the differential emission measure $D(T)$ in such a way that the total emission measure as integrated over that interval as well as the emission-measure weighted temperature remain constant. The resulting spectra are almost indistinguishable. This point is illustrated in Fig. 16.

There are several methods to reconstruct $D(T)$. We refer here to Kaastra et al. (1996a) for more details. All these methods have been implemented in SPEX. They have been applied to stellar coronae or Solar spectra, but recently also to clusters of galaxies. See for example Kaastra et al. (2004). In the last case, an analytical power-law approximation $D(T) \sim T^{1 / \alpha}$ has been used.

Also for photo-ionised plasmas a similar technique can be applied. Instead of the temperature $T$, one uses the ionisation parameter $\xi$ (XSTAR users) or $U$ (Cloudy users). In some cases (like Seyfert 2 galaxies with emission spectra) one recovers $D(\xi)$ from the observed emission spectra; in other cases (like Seyfert 1 galaxies with absorption spectra), a continuum model is applied to the observed spectrum, and $D(\xi)$ is recovered from the absorption features in the spectrum. Most of what has been said before on $D(T)$ also applies to this case, i.e. the resolution in $\xi$ and the various methods to reconstruct the continuous distribution $D(\xi)$.

Acknowledgements The authors thank ISSI (Bern) for support of the team "Non-virialised X-ray components in clusters of galaxies". SRON is supported financially by NWO, the Netherlands Organisation for Scientific Research. 


\section{References}

E. Anders, N. Grevesse, Geochim. Cosmochim. Acta 53, 197 (1989)

K.A. Arnaud, ASP Conf. Ser. 101, 17 (1996)

M. Arnaud, R. Rothenflug, Astron. Astrophys. Suppl. Ser. 60, 425 (1985)

A. Bhardwaj, R.F. Elsner, G.R. Gladstone, T.E. Cravens, C.M. Lisse, et al., Planet. Space Sci. 55, 1135 (2006)

E. Churazov, M. Haehnelt, O. Kotov, R. Sunyaev, Mon. Not. R. Astron. Soc. 323, 93 (2001)

G.J. Ferland, K.T. Korista, D.A. Verner et al., Publ. Astron. Soc. Pac. 110, 761 (1998)

M.R. Gil'fanov, R.A. Syunyaev, E.M. Churazov, Sov. Astron. Lett. 13, 3 (1987)

G. Herzberg, Atomic Spectra and Atomic Structure, 2nd edn. (Dover, New York, 1944)

J.S. Kaastra, R. Mewe, Astron. Astrophys. Suppl. Ser. 97, 443 (1993)

J.S. Kaastra, R. Mewe, D.A. Liedahl, Astron. Astrophys. 314, 547 (1996a)

J.S. Kaastra, R. Mewe, H. Nieuwenhuijzen, in UV and X-ray Spectroscopy of Astrophysical and Laboratory Plasmas, ed. by K. Yamashita, T. Watanabe (Univ. Ac. Press, Tokyo, 1996b), p. 411

J.S. Kaastra, T. Tamura, J.R. Peterson et al., Astron. Astrophys. 413, 415 (2004)

T.R. Kallman, R. McCray, Astrophys. J. Suppl. Ser. 50, 263 (1982)

W.J. Karzas, R. Latter, Astrophys. J. Suppl. Ser. 6, 167 (1961)

K.D. Kuntz, S.L. Snowden, Astrophys. J. 543, 195 (2000)

E. Landi, G. Del Zanna, P.R. Young et al., Astrophys. J. Suppl. Ser. 162, 261 (2006)

M. Landini, B.C. Monsignori Fossi, Astron. Astrophys. 6, 468 (1970)

K. Lodders, Astrophys. J. 591, 1220 (2003)

W. Lotz, Z. Physik 216, 441 (1968)

U. Maio, K. Dolag, B. Ciardi, L. Tornatore, Mon. Not. R. Astron. Soc. 379, 963 (2007)

R. Mewe, Sol. Phys. 22, 459 (1972)

R. Mewe, in X-ray Spectroscopy in Astrophysics. Lect. Notes in Phys., vol. 520 (1999), p. 109

R. Mewe, J.R. Lemen, G.H.J. van den Oord, Astron. Astrophys. Supp. 65, 511 (1986)

R. Mewe, J.S. Kaastra, D.A. Liedahl, Legacy 6, 16 (1995)

V. Petrosian, A. Bykov, Y. Rephaeli, Space Sci. Rev. (2008). doi:10.1007/s11214-008-9327-2

J.C. Raymond, AIP Conf. Proc. 774, 15 (2005)

J.C. Raymond, B.W. Smith, Astrophys. J. Suppl. Ser. 35, 419 (1977)

P. Richter, F. Paerels, J.S. Kaastra, Space Sci. Rev. (2008). doi:10.1007/s11214-008-9325-4

M. Sako, S.M. Kahn, E. Behar et al., Astron. Astrophys. 365, L168 (2001)

M.J. Seaton, Mon. Not. R. Astron. Soc. 119, 81 (1959)

R.K. Smith, N.S. Brickhouse, D.A. Liedahl, J.C. Raymond, Astrophys. J. 556, L91 (2001)

R.S. Sutherland, M.A. Dopita, Astrophys. J. Suppl. Ser. 88, 253 (1993)

J. Vink, J.M. Laming, M.F. Gu, A. Rasmussen, J.S. Kaastra, Astrophys. J. 587, L31 (2003)

J. Wilms, A. Allen, R. McCray, Astrophys. J. 542, 914 (2000)

Y. Yao, Q.D. Wang, Astrophys. J. 641, 930 (2006) 